\title{
Harmonic Maps with Fixed Singular Sets
}

\author{
ROBERT HARDT* and LIBIN MOU
}

\section{$\S$ 0. Introduction}

Here, for a smooth domain $\Omega$ in $\mathbf{R}^{m}$ and a compact smooth Riemannian manifold $N$ we study a space $\mathcal{H}$ consisting of all harmonic maps $u: \Omega \rightarrow N$ that have a singular set being a fixed compact subset $Z$ of $\Omega$ having finite $m-3$ dimensional Minkowski content. This holds if, for example, $Z$ is $m-3$ rectifiable [F, 3.2.14]. We define a suitable topology on $\mathcal{H}$ using Hölder norms on derivatives weighted by powers of the distance to $Z$.

We study the structure of $\mathcal{H}$ and other properties, such as stability and minimality, under perturbations. For example, near a homogeneous harmonic map that is singular only at the origin, $\mathcal{H}$ is, for suitable powers of the weights, a smooth manifold [Theorem 4.7]. An application [Theorem 5.6] of the result to some well-known harmonic maps, such as the homogeneous extension of the harmonic maps from $\mathbf{S}^{2}$ to $\mathbf{S}^{2}$, the identity from $\mathbf{S}^{m-1}$ to itself and the equator maps from $\mathbf{S}^{m-1}$ to $\mathbf{S}^{m}$, gives that their boundary data can be perturbed in any directions of the eigenvectors corresponding to the positive eigenvalues (of the boundary data) to obtain new harmonic maps. These new harmonic maps are also stable (energy minimizing, unique) if the perturbed maps are strictly stable (strictly minimizing), which is implied by Theorems 3.5, 3.8 saying that stability and minimality are preserved under small perturbations.

For smooth harmonic maps, we prove that $\mathcal{H}$ is a Banach manifold modelled on the space of boundary data, and the projection map that sends $u$ to $u \mid \partial \Omega$ is Fredholm of index 0 [Theorem 6.4]. Locally, near $u \in \mathcal{H}$ with $K_{0}$ being the space of smooth Jacobi fields along $u$ that vanish on $\partial \Omega$, some neighborhood of $u$ in $\mathcal{H}$ is diffeomorphic to a submanifold (of codimension $\operatorname{dim}\left(K_{0}\right)$ ) of the product of $K_{0}$ with the space of the boundary data [Theorem 6.2]. Using the global structure theorem, we prove a generic uniqueness property of smooth harmonic maps [Theorem 6.8], which establishes the first category nature of the set of all boundary maps that occur as trace of two distinct harmonic maps having same energy.

We prove a Schauder-type estimate and a pointwise estimate in Section 2 [Theorems 2.1, 2.3]. This is relevant for a study of the Fredholm property of the Jacobi operator, which is essential to apply the implicit function theorem. For isolated singularities, a very detailed analysis of this property with perturbation applications has recently been given by N. Smale [SN3].

If one allows the singularity to vary, there is the example of [HKL] where the boundary data corresponds under the sterographic projection to the conformal map $z^{2}$. Here there is a one parameter family of distinct harmonic maps having this Dirichlet boundary data and the same energy (by energy minimality). The singularity varies. This phenomenon holds for any $n$-axially symmetric boundary data of non-zero degree when $n \geq 2$. (See $[\mathbf{H K L}]$.)

For smooth case, we follow with some modifications some steps in Brian White's study [WB] of the corresponding theory for smooth immersed minimal submanifolds. The presence of singularities demands various new estimates and leads to several interesting examples of singular Jacobi fields and families of harmonic maps. The authors would like to thank Frank Pacard and Nat Smale for their interest and for pointing out an error in an earlier version of this paper.

\section{$\S$ 1. Preliminaries}

Appeared in Journal of Geometric Analysis. Vol. 2, 5 (1992) 445-488. 
1.1. Definitions. Here we assume that $\Omega \subseteq \mathbf{R}^{m}(m \geq 3)$ is a smooth bounded domain with standard metric, and $N \subseteq \mathbf{R}^{p}(p \geq 2)$ is a smooth compact submanifold (without boundary). All our discussions can be modified to hold with $\Omega$ replaced by a compact Riemannian manifold with boundary. Let

$$
H^{1}(\Omega, N)=\left\{u \in H^{1}\left(\Omega, \mathbf{R}^{p}\right): u(x) \in N \text { for a.e. } x \in \Omega\right\}
$$

where $H^{1}\left(\Omega, \mathbf{R}^{p}\right)$ is the set of $u \in L^{2}\left(\Omega, \mathbf{R}^{p}\right)$ whose distributional gradient is square integrable. The energy of $u \in H^{1}\left(\Omega, \mathbf{R}^{p}\right)$ is denoted

$$
E(u)=\int_{\Omega}|\nabla u|^{2} .
$$

Now we introduce the spaces for maps that are possibly singular (i.e., discontinuous) on a fixed subset of $\Omega$. Let $Z$ be a compact subset of $\Omega$ having finite $m-q(q \geq 0)$ dimensional Minkowski content $\mathcal{M}^{m-q}(Z)$ [F, 3.2.37]. For $x \in \Omega$, denote by $\rho(x)=\operatorname{dist}(x, Z)$, and for $r>0$, denote

$$
Z_{r}=\left\{z \in \mathbf{R}^{m}: \rho(z)<r\right\} ; \quad Z_{r, 2 r}=Z_{2 r} \backslash \bar{Z}_{r} ; \quad \Omega_{0}=\Omega \backslash Z ; \quad \Omega_{r}=\Omega \backslash \bar{Z}_{r} .
$$

Take a number $r_{0}>0$ so that $Z_{3 r_{0}} \subseteq \Omega$. For an integer $k \geq 0,0 \leq \alpha<1$ and $\nu \in \mathbf{R}$, we define a norm $\|u\|_{k, \alpha ; \nu}$ for $u \in C_{\mathrm{loc}}^{k, \alpha}\left(\Omega_{0}, \mathbf{R}^{p}\right)$ :

$$
\|u\|_{k, \alpha ; \nu}=\sup _{0<r \leq r_{0}} \sum_{\beta \in\{0, \alpha\}} \sum_{j=0}^{k} r^{j+\beta-\nu}\left\|\nabla^{j} u\right\|_{(\beta), Z_{r, 2 r}}+\|u\|_{k, \alpha ; \Omega_{r_{0}}}
$$

where $\|\cdot\|_{k, \alpha, \Sigma}=\|\cdot\|_{C^{k, \alpha}(\Sigma)}$ is Hölder norm on $\Sigma$. In particular, $\|\cdot\|_{(\alpha), \Sigma}=\|\cdot\|_{C^{0, \alpha}(\Sigma)}$ and $\|\cdot\|_{(0), \Sigma}=\|\cdot\|_{C^{0}(\Sigma)}$.

Roughly speaking, $\|u\|_{k, \alpha ; \nu}<\infty$ if and only if $u, \rho \nabla u, \ldots, \rho^{k} \nabla^{k} u$ are bounded by $\rho^{\nu}$. This norm allows $u$ to be singular on $Z$, yet gives control of the asymptotic behavior of $u$ near $Z$. It is used in [CHS] to construct the first example of minimal surface with singularity that is not a cone. In [SN1] and [ML1], it is used to prove some bridge principles, according to which a minimal surface or a harmonic map with prescribed finite singular set can be constructed from those with single singularities.

Using this norm, we define the following spaces:

$$
\begin{gathered}
C^{k, \alpha ; \nu}\left(\Omega_{0}, \mathbf{R}^{p}\right)=\left\{u \in C_{\mathrm{loc}}^{k, \alpha}\left(\Omega_{0}, \mathbf{R}^{p}\right):\|u\|_{k, \alpha ; \nu}<\infty\right\} ; \\
C_{0}^{k, \alpha ; \nu}\left(\Omega_{0}, \mathbf{R}^{p}\right)=\left\{u \in C^{k, \alpha ; \nu}\left(\Omega_{0}, \mathbf{R}^{p}\right): u=0 \text { on } \partial \Omega\right\} ; \\
C^{k, \alpha ; \nu}\left(\Omega_{0}, N\right)=\left\{u \in C^{k, \alpha ; \nu}\left(\Omega_{0}, \mathbf{R}^{p}\right): u(x) \in N \text { for } x \in \Omega_{0}\right\} .
\end{gathered}
$$

For $u \in C^{k, \alpha ; \nu}\left(\Omega_{0}, N\right)$, we define sets of vector fields along $u$ :

$$
\begin{aligned}
& C^{k, \alpha ; \nu}\left(\Omega_{0}, T_{u} N\right)=\left\{\kappa \in C^{k, \alpha ; \nu}\left(\Omega_{0}, \mathbf{R}^{p}\right): \kappa \in T_{u} N\right\} ; \\
& C_{0}^{k, \alpha ; \nu}\left(\Omega_{0}, T_{u} N\right)=\left\{\kappa \in C_{0}^{k, \alpha ; \nu}\left(\Omega_{0}, \mathbf{R}^{p}\right): \kappa \in T_{u} N\right\},
\end{aligned}
$$

where $\kappa \in T_{u} N$ means that $\kappa(x) \in T_{u(x)} N$ for $x \in \Omega_{0}$. Similarly $\kappa \perp T_{u} N$ means that $\kappa(x) \perp T_{u(x)} N$ for $x \in \Omega_{0}$.

We will also use the following spaces of boundary data:

$$
\begin{aligned}
C^{k, \alpha}\left(\partial \Omega, \mathbf{R}^{p}\right) & =\left\{\varphi: \varphi=u \mid \partial \Omega, u \in C^{k, \alpha}\left(\Omega, \mathbf{R}^{p}\right)\right\} ; \\
C^{k, \alpha}(\partial \Omega, N) & =\left\{\varphi \in C^{k, \alpha}\left(\partial \Omega, \mathbf{R}^{p}\right): \varphi(x) \in N \text { for } x \in \partial \Omega\right\} ; \\
C^{k, \alpha}\left(\partial \Omega, T_{\psi} N\right) & =\left\{\kappa \in C^{k, \alpha}\left(\partial \Omega, \mathbf{R}^{p}\right): \kappa \in T_{\psi} N\right\},
\end{aligned}
$$

where $\psi \in C^{k, \alpha}(\partial \Omega, N)$. Here the norm $\|\cdot\|_{k, \alpha, \partial \Omega}$ on $C^{k, \alpha}\left(\partial \Omega, \mathbf{R}^{p}\right)$ is defined by

$$
\|\varphi\|=\inf \left\{\|u\|_{k, \alpha, \Omega}: u \mid \partial \Omega=\varphi, u \in C^{k, \alpha}\left(\Omega, \mathbf{R}^{p}\right)\right\} .
$$

Note that $C^{k, \alpha ; 0}\left(\Omega_{0}, N\right)$ contains many interesting maps. For example, $C^{k, \alpha ; 0}\left(\mathbf{B}^{m} \backslash\{0\}, N\right)$ contains the homogeneous extensions of smooth maps from $\mathbf{S}^{m-1}$ to $N$. 
1.2.

(a). Note that $C^{k, \alpha}(\Omega, N), C^{k, \alpha}(\partial \Omega, N)$ and $C^{k, \alpha ; \nu}\left(\Omega_{0}, N\right)(\nu \geq 0)$ are Banach manifolds, modelled on their tangent spaces:

$$
\begin{aligned}
& T_{u} C^{k, \alpha}(\Omega, N)=C^{k, \alpha}\left(\Omega, T_{u} N\right) \quad \text { at } u \in C^{k, \alpha}(\Omega, N) ; \\
& T_{u} C^{k, \alpha ; \nu}\left(\Omega_{0}, N\right)=C^{k, \alpha ; \nu}\left(\Omega_{0}, T_{u} N\right) \quad \text { at } u \in C^{k, \alpha ; \nu}\left(\Omega_{0}, N\right) ; \\
& T_{\psi} C^{k, \alpha}(\partial \Omega, N)=C^{k, \alpha}\left(\partial \Omega, T_{\psi} N\right) \quad \text { at } \psi \in C^{k, \alpha}(\partial \Omega, N),
\end{aligned}
$$

which are Banach spaces. For example, one can check that a neighborhood of $u$ in $C^{k, \alpha ; \nu}\left(\Omega_{0}, N\right)(\nu \geq 0)$ is diffemorphic to a neighborhood of 0 in $C^{k, \alpha ; \nu}\left(\Omega_{0}, T_{u} N\right)$ under the exponential map

$$
\exp _{u}: C^{k, \alpha ; \nu}\left(\Omega_{0}, T_{u} N\right) \rightarrow C^{k, \alpha ; \nu}\left(\Omega_{0}, N\right)
$$

defined by

$$
\left(\exp _{u} \kappa\right)(x)=\exp _{u(x)} \kappa(x) \text { for } \kappa \in C^{k, \alpha ; \nu}\left(\Omega_{0}, T_{u} N\right) .
$$

(b). $C^{k, \alpha}\left(\Omega, \mathbf{R}^{p}\right) \subseteq C^{k, \alpha ; 0}\left(\Omega_{0}, \mathbf{R}^{p}\right)$ with equality valid only when $Z=\emptyset$. If $k \leq k^{\prime}, \alpha \leq \alpha^{\prime}$ and $\nu \leq \nu^{\prime}$, then $C^{k^{\prime}, \alpha^{\prime} ; \nu^{\prime}}\left(\Omega_{0}, \mathbf{R}^{p}\right) \subseteq C^{k, \alpha ; \nu}\left(\Omega_{0}, \mathbf{R}^{p}\right)$. The inclusion is compact if $k+\alpha<k^{\prime}+\alpha^{\prime}$ and $\nu<\nu^{\prime}$.

(c). If $u \in C^{k, \alpha ; \nu}\left(\Omega_{0}, \mathbf{R}^{p}\right)$, then $\frac{\partial^{\beta}}{\partial x^{\beta}} u \in C^{k-|\beta|, \alpha ; \nu-|\beta|}\left(\Omega_{0}, \mathbf{R}^{p}\right)$ for $|\beta| \leq k$.

If $u \in C^{k, \alpha ; \nu}\left(\Omega_{0}, \mathbf{R}^{p}\right)$ and $v \in C^{k, \alpha ; \mu}\left(\Omega_{0}, \mathbf{R}^{p}\right)$, then the product $u \cdot v \in C^{k, \alpha ; \nu+\mu}\left(\Omega_{0}, \mathbf{R}\right)$.

As a counterpart of $C^{k, \alpha ; \nu}\left(\Omega_{0}, N\right)$, we define $H^{k ; \delta}\left(\Omega, \mathbf{R}^{p}\right)$ with a norm $\|u\|_{k ; \delta}$ for $\delta \in \mathbf{R}$ and integer $k \geq 0$ as follows.

$$
\begin{gathered}
\|u\|_{k ; \delta}=\sum_{j=1}^{k}\left\|\rho^{j-\delta-1} \nabla^{j} u\right\|_{L^{2}(\Omega)} . \\
H^{k ; \delta}\left(\Omega, \mathbf{R}^{p}\right)=\left\{u \in L^{2}\left(\Omega, \mathbf{R}^{p}\right):\|u\|_{k ; \delta}<\infty\right\}
\end{gathered}
$$

We have the following

1.3. Lemma. Suppose that $Z \subseteq \Omega$ has finite $m-q$ dimensional Minkowski content $\mathcal{M}^{m-q}(Z), k \geq 0$, $\nu \in \mathbf{R}$ and $0 \leq \alpha<1$. Then

$$
C^{k, \alpha ; \nu}\left(\Omega_{0}, \mathbf{R}^{p}\right) \subseteq H^{k ; \delta}\left(\Omega, \mathbf{R}^{p}\right)
$$

for $\delta<\nu+\frac{q-2}{2}$.

In particular, if $q>2$ and $\nu \geq 0$, then $C^{k, \alpha ; \nu}\left(\Omega_{0}, \mathbf{R}^{p}\right) \subset H^{1 ; 0}\left(\Omega, \mathbf{R}^{p}\right) \subset H^{1}\left(\Omega, \mathbf{R}^{p}\right)$.

Proof : We claim that if $\rho(x)=\operatorname{dist}(x, Z)$ and $\nu+q>0$, then

$$
\rho^{\nu} \in L^{1}(\Omega) \text {. }
$$

To prove (1.3.1), it suffices to show that $\rho^{\nu} \in L^{1}\left(Z_{r_{0}}\right)$ for small $r_{0}$. Indeed, that $\mathcal{M}^{m-q}(Z)$ is finite implies that for some constant $C_{1}$ and small $r_{0}, \mathcal{L}^{m}\left(Z_{r}\right) \leq C_{1} r^{q}$ when $0<r<r_{0}$. Thus we have

$$
\begin{aligned}
\int_{Z_{r_{0}}} \rho^{\nu} d x & =\sum_{i=0}^{\infty} \int_{Z_{2^{-i-1} r_{0}, 2^{-i} r_{0}}} \rho^{\nu} d x \\
& \leq \sum_{i=0}^{\infty}\left(\left(r_{0} 2^{-i-1}\right)^{\nu}+\left(r_{0} 2^{-i}\right)^{\nu}\right)\left(r_{0} 2^{-i}\right)^{q} \\
& =\left(1+2^{-\nu}\right) r_{0}^{\nu+q} \sum_{i=0}^{\infty}\left(2^{\nu+q}\right)^{-i} \\
& =\frac{\left(1+2^{-\nu}\right) r_{0}^{\nu+q}}{1-2^{-\nu-q}}<\infty .
\end{aligned}
$$

Now that $\delta<\nu+\frac{q-2}{2}$ implies that if $u \in C^{k, \alpha ; \nu}\left(\Omega_{0}, T_{u} N\right)$, then for $j=0,1, \ldots, k,\left|\rho^{j-\delta-1} \nabla^{j} u\right|^{2} \leq$ $C \rho^{2(\nu-\delta-1)} \in L^{1}$. By definition, then $u \in H^{k ; \delta}\left(\Omega, \mathbf{R}^{p}\right)$. 
In the first and second variational formulae that follow, we assume that $k \geq 2,0 \leq \alpha<1$ and $\nu \geq 0$.

\subsection{First variation formula of energy.}

Since $N$ is smooth, we may take a $\delta=\delta(N)>0$ such that for any

$$
x \in N_{\delta} \equiv\left\{x \in \mathbf{R}^{p}: \operatorname{dist}(x, N) \leq \delta\right\}
$$

there exists a unique point $\pi(x) \in N$ such that $\operatorname{dist}(x, N)=|x-\pi(x)|$. Furthermore, for $y \in N$,

$$
D \pi(y)=P_{y},
$$

the projection map from $\mathbf{R}^{p}$ to $T_{y} N$ [HL1].

Suppose $u \in C^{k, \alpha ; 0}\left(\Omega_{0}, N\right), \kappa \in H^{1}\left(\Omega, T_{u} N\right) \cap L^{\infty}$ and $u_{t}=\pi(u+t \kappa):(-\varepsilon, \varepsilon) \rightarrow H^{1}(\Omega, N)$ is a differentiable curve in $H^{1}(\Omega, N)$ with $u=u_{0},\left.\frac{d}{d t}\right|_{t=0} u_{t}=D \pi(u) \kappa=P_{u} \kappa=\kappa \in T_{u} N$, then the first variation formula is

$$
\left.\frac{d}{d t}\right|_{t=0} E\left(u_{t}\right)=2 \int_{\Omega}-H(u) \cdot \kappa+\int_{\partial \Omega} \frac{\partial u}{\partial \mathbf{n}} \cdot \kappa,
$$

where

$$
H(u)=P_{u} \Delta u=\Delta u-A(u)(\nabla u, \nabla u),
$$

$\mathbf{n}$ is the outward normal unit direction of $\partial \Omega, A(u)$ is the second fundamental form of $N$, evaluated at $u$, and $\nabla u=\left(\frac{\partial u}{\partial x_{1}}, \ldots, \frac{\partial u}{\partial x_{m}}\right)$. Note that $\frac{\partial u}{\partial x_{i}} \in T_{u} N$ for each $i$. Also $H(u) \in C^{k-2, \alpha ;-2}\left(\Omega_{0}, T_{u} N\right)$ and

$$
H: C^{k, \alpha ; 0}\left(\Omega_{0}, N\right) \rightarrow C^{k-2, \alpha ;-2}\left(\Omega_{0}, \mathbf{R}^{p}\right)
$$

is a smooth map (between the two Banach spaces).

A map $u \in H^{1}(\Omega, N)$ is a harmonic map if it is a solution of Euler-Lagrange equation

$$
H(u)=0 .
$$

In this paper we are interested in set of harmonic maps in $C^{k, \alpha ; 0}\left(\Omega_{0}, N\right)$, which we denote by

$$
\mathcal{H}=\left\{u \in C^{k, \alpha ; 0}\left(\Omega_{0}, N\right): u \text { is harmonic }\right\} .
$$

1.5. Remarks .

(a). $H(u)$ can be viewed as the derivative of $E(u)$ : For $\kappa \in H^{1}\left(\Omega, T_{u} N\right) \cap L^{\infty}$,

$$
\langle\kappa, D E(u)\rangle=\int_{\Omega}-H(u) \cdot \kappa=\langle-H(u), \kappa\rangle_{L^{2}} .
$$

(b). Also $H(u)=$ Trace $(\nabla d u)$, where $\nabla d u$ is the bilinear form defined by $\nabla d u(\kappa, \eta)=\left(\nabla_{\kappa} d u\right)(\eta)$. See $[\mathbf{E L}][\mathbf{J}]$.

(c). $H(u)$ can also be described in terms of local coordinates of $N$. See $[\mathbf{J}]$, for example.

1.6. Second variational formula of energy. Suppose that $u \in C^{k, \alpha ; 0}\left(\Omega_{0}, N\right), \kappa, \eta \in H^{1}\left(\Omega, T_{u} N\right) \cap L^{\infty}$ and

$$
u_{s t}:(-\varepsilon, \varepsilon) \times(-\varepsilon, \varepsilon) \rightarrow H^{1}(\Omega, N)
$$

(e.g., $\left.u_{s t}=\pi(u+t \kappa+s \eta)\right)$ is a differentiable 2 -parameter variation with

$$
(\kappa, \eta)=\left.\left(\frac{\partial}{\partial t} u_{s t}, \frac{\partial}{\partial s} u_{s t}\right)\right|_{s=t=0} .
$$


Then the second variation is

$$
\left.\frac{\partial^{2}}{\partial t \partial s}\right|_{s=t=0} E\left(u_{s t}\right)=2 \int_{\Omega}-J_{u} \kappa \cdot \eta+2 \int_{\partial \Omega} \frac{\partial \kappa}{\partial \mathbf{n}} \cdot \eta,
$$

where $J_{u}=P_{u} \circ D H(u)$ is given by

$$
J_{u} \kappa=\Delta \kappa-D A(u)(\kappa, \nabla u, \nabla u)-2 A(u)(\nabla \kappa, \nabla u)+A(u)(H(u), \kappa) .
$$

1.7. $J_{u}$ is called the Jacobi operator with respect to $u$. Note that $J_{u}$ maps $C^{k, \alpha ; \nu}\left(\Omega_{0}, T_{u} N\right)$ to $C^{k-2, \alpha ; \nu-2}\left(\Omega_{0}, T_{u} N\right)$, and it is linear and uniformly elliptic. A solution $\kappa \in C^{k, \alpha ; \nu}\left(\Omega_{0}, T_{u} N\right)$ of $J_{u} \kappa=0$ is called a Jacobi field in $C^{k, \alpha ; \nu}\left(\Omega_{0}, T_{u} N\right)$ with respect to $u$. Furthermore, the symmetry of $\kappa$ and $\eta$ in (1.6.1) implies that $J_{u}$ is self-adjoint; that is, for all $\kappa, \eta \in H_{0}^{1}\left(\Omega, T_{u} N\right) \cap L^{\infty}$,

$$
\left\langle J_{u} \kappa, \eta\right\rangle_{L^{2}}=\left\langle\kappa, J_{u} \eta\right\rangle_{L^{2}}=-\int_{\Omega} \nabla \kappa \cdot \nabla \eta+D A(\kappa, \nabla u, \nabla u) \cdot \eta .
$$

If $u$ is harmonic, then

$$
J_{u} \kappa=\langle\kappa, D H(u)\rangle=\Delta \kappa-D A(u)(\kappa, d u, d u)-2 A(u)(d \kappa, d u) .
$$

If $u$ is harmonic, then for $\kappa \in H^{1}\left(\Omega, T_{u} N\right) \cap L^{\infty}$,

$$
J_{u} \kappa=\langle\kappa, D H(u)\rangle=\lim _{t \rightarrow 0} \frac{1}{t}[H(\pi(u+t \kappa))-H(u)] .
$$

This extends the definition of $J_{u}$ to $H^{1}\left(\Omega, \mathbf{R}^{p}\right)$ so that $J_{u} \kappa=0$ for $\kappa \perp T_{u} N$.

$J_{u}$ may have different forms. See $[\mathbf{J}]$, for example.

\section{$\S 2$. Some Estimates for Jacobi Fields}

To use implicit function theorem to study the solutions of $H(u)=0$, a key step is to show the Fredholm property of the linearization of $H$, (i.e., the Jacobi operator $J_{u}$ ). For this purpose and other possible applications, we prove an estimate of Schauder's type and a pointwise bound estimate for solutions in $C^{k, \alpha ; \nu}\left(\Omega_{0}, \mathbf{R}^{p}\right)$ of elliptic systems whose coefficients may have singularities.

We will use the notations introduced in Section 1, with $k \geq 2,0<\alpha<1$ and $\nu \in \mathbf{R}$.

2.1. Theorem. Suppose $\kappa \in C_{\mathrm{loc}}^{k, \alpha}\left(\Omega_{0}, \mathbf{R}^{p}\right)$ and $J$ is a linear elliptic operator in the following form:

$$
\begin{aligned}
& J u=\left(\sum_{j=1}^{p} J_{i j}(\cdot, D) u^{j}\right)_{1 \leq i \leq p}, \\
& J_{i j}(x, D)=\sum_{s t} a_{i j}^{s t}(x) D_{s} D_{t}+\sum_{s} b_{i j}^{s}(x) D_{s}+c_{i j}(x),
\end{aligned}
$$

where the coefficients satisfy the following:

$$
\begin{gathered}
a=\left(a_{i j}^{s t}\right) \in C^{k-2, \alpha ; 0}\left(\Omega_{0}, \mathbf{R}^{p^{2} \times m^{2}}\right), \quad b=\left(b_{i j}^{s}\right) \in C^{k-2, \alpha ;-1}\left(\Omega_{0}, \mathbf{R}^{p^{2} \times m}\right), \\
c=\left(c_{i j}\right) \in C^{k-2, \alpha ;-2}\left(\Omega_{0}, \mathbf{R}^{p^{2}}\right), \quad J \kappa \in C^{k-2, \alpha ; \nu-2}\left(\Omega_{0}, \mathbf{R}^{p}\right), \\
J(x, \xi)=\operatorname{det}\left(\sum_{s t} a_{i j}^{s t} \xi_{s} \xi_{t}\right)_{p \times p} \geq \lambda|\xi|^{2 p}
\end{gathered}
$$


for all $\xi \in \mathbf{R}^{m}$ and some constant $\lambda>0$.

Then there exists a constant $C$ depending only $\|a\|_{k-2, \alpha ; 0},\|b\|_{k-2, \alpha ;-1},\|c\|_{k-2, \alpha ;-2}, \nu, m, p, \lambda$ and $\Omega$ such that

$$
\|\kappa\|_{k, \alpha ; \nu} \leq C\left[\|J \kappa\|_{k-2, \alpha ; \nu-2}+\|\kappa\|_{k-2,0 ; \nu}+\|\kappa\|_{k, \alpha, \partial \Omega}\right] .
$$

Proof : For $0<r<r_{0}$, and $x_{0} \in Z_{r, 2 r}$, we apply the Schauder's interior estimate on the ball $\mathbf{B}_{R}=\mathbf{B}_{R}\left(x_{0}\right)$ with $R=2 r / 3$. To be specific, let $\sigma \in \mathbf{R}$ and consider the following norms:

$$
\|\kappa\|_{k, \alpha ; \mathbf{B}_{R}}^{(\sigma)}=\sum_{j=0}^{k} \sup _{x \in \mathbf{B}_{R}} d_{x}^{j+\sigma}\left|\nabla^{j} \kappa(x)\right|+\sum_{j=0}^{k} \sup _{x, y \in \mathbf{B}_{R}} d_{x y}^{j+\alpha+\sigma} \frac{\left|\nabla^{j} \kappa(x)-\nabla^{j} \kappa(y)\right|}{|x-y|^{\alpha}}
$$

$$
{ }^{*}\|\kappa\|_{k, \alpha ; \mathbf{B}_{R}}^{(\sigma)}=\sum_{\beta \in\{0, \alpha\}} \sum_{j=0}^{k} R^{j+\beta+\sigma}\left\|\nabla^{j} \kappa\right\|_{(\beta), \mathbf{B}_{R}}
$$

where $d_{x}=\operatorname{dist}\left(x, \partial \mathbf{B}_{R}\right)$ and $d_{x y}=\min \left\{d_{x}, d_{y}\right\}$. Here $\|\cdot\|_{k, \alpha ; \mathbf{B}_{R}}^{(\sigma)}$ is equivalent to the norm $|\cdot|_{k, \alpha ; \mathbf{B}_{R}}^{(\sigma)}$ defined in [GT, $\S 6.1]$. When $\sigma \geq 0$, the following simple relation holds

$$
{ }^{*}\|\kappa\|_{k, \alpha ; \mathbf{B}_{R / 2}}^{(\sigma)} \leq\|\kappa\|_{k, \alpha ; \mathbf{B}_{R}}^{(\sigma)} \leq^{*}\|\kappa\|_{k, \alpha ; \mathbf{B}_{R}}^{(\sigma)} \leq\|\kappa\|_{k, \alpha ;-\sigma} .
$$

(where $\|\kappa\|_{k, \alpha ;-\sigma}$ is the norm defined in 1.1) In particular, we have

$$
\begin{aligned}
\|a\|_{k-2, \alpha ; \mathbf{B}_{R}}^{(0)} & \leq\|a\|_{k-2, \alpha ; 0}, \\
\|b\|_{k-2, \alpha ; \mathbf{B}_{R}}^{(1)} & \leq\|b\|_{k-2, \alpha ;-1}, \\
\|c\|_{k-2, \alpha ; \mathbf{B}_{R}}^{(2)} & \leq\|c\|_{k-2, \alpha ;-2} .
\end{aligned}
$$

Thus the Schauder's interior estimates [GT , Theorem 6.2] ([DN, Theorem 1] for systems) imply

$$
\|\kappa\|_{k, \alpha ; \mathbf{B}_{R}}^{(0)} \leq C_{1}\left[\|J \kappa\|_{k-2, \alpha ; \mathbf{B}_{R}}^{(2)}+\|\kappa\|_{0, \mathbf{B}_{R}}\right]
$$

where $C_{1}$, and $C_{2}, C_{3} \ldots$ in the sequel, are constants as stated in the theorem. Combined with (2.1.4), (2.1.5) gives

$$
\sum_{\beta \in\{0, \alpha\}} \sum_{j=0}^{k} r^{j+\beta}\left\|\nabla^{j} \kappa\right\|_{(\beta), \mathbf{B}_{r / 3}} \leq C_{2}\left[\sum_{\beta \in\{0, \alpha\}} \sum_{j=0}^{k-2} r^{j+\beta+2}\left\|\nabla^{j} J \kappa\right\|_{(\beta), \mathbf{B}_{2 r / 3}}+\|\kappa\|_{0, \mathbf{B}_{2 r / 3}}\right] .
$$

Multiplying (2.1.6) by $r^{-\nu}$, we get

$$
\begin{aligned}
& \sum_{\beta \in\{0, \alpha\}} \sum_{j=0}^{k} r^{j+\beta-\nu}\left\|\nabla^{j} \kappa\right\|_{(\beta), \mathbf{B}_{r / 3}} \\
& \leq C_{2}\left[\sum_{\beta \in\{0, \alpha\}} \sum_{j=0}^{k-2} r^{j+\beta-(\nu-2)}\left\|\nabla^{j} J_{u} \kappa\right\|_{(\beta), \mathbf{B}_{2 r / 3}}+r^{-\nu}\|\kappa\|_{0, \mathbf{B}_{2 r / 3}}\right], \\
& \leq C_{3}\left[\|J \kappa\|_{k-2, \alpha ; \nu-2}+\|\kappa\|_{k-2,0 ; \nu}\right] .
\end{aligned}
$$


Now we apply the fact that any function $\eta \in C^{\alpha}\left(\Omega_{0}\right)$ satisfies

$$
\|\eta\|_{\alpha, Z_{r, 2 r}} \leq 2 \cdot 3^{\alpha} \sup _{x \in Z_{r, 2 r}}\left[r^{-\alpha}\|\eta\|_{0, \mathbf{B}_{r / 3}(x)}+\|\eta\|_{(\alpha), \mathbf{B}_{r / 3}(x)}\right]
$$

to $\nabla^{j} \kappa(j=0, \cdots, k)$, and use $(2.1 .7)$, to get

$$
\begin{aligned}
& \sum_{\beta \in\{0, \alpha\}} \sum_{j=0}^{k} r^{j+\beta-\nu}\left\|\nabla^{j} \kappa\right\|_{(\beta), Z_{r, 2 r}} \\
& \leq C_{4} \sup _{x \in Z_{r, 2 r}} \sum_{\beta \in\{0, \alpha\}} \sum_{j=0}^{k} r^{j+\beta-\nu}\left\|\nabla^{j} \kappa\right\|_{(\beta), \mathbf{B}_{r / 3}(x)} \\
& \leq C_{5}\left[\|J \kappa\|_{k-2, \alpha ; \nu-2}+\|\kappa\|_{k-2,0 ; \nu}\right] .
\end{aligned}
$$

To estimate $\|\kappa\|_{k, \alpha, \Omega_{r_{0}}}$, we apply the Schauder estimates $\left[\mathbf{A D N}\right.$, Theorem 9.3], to $\Omega_{r_{0}} \subset \subset \Omega_{r_{0} / 2}$ (with $\mathcal{D}=\Omega_{r_{0} / 2}, \mathcal{A}=\Omega_{r_{0}}$ and $\Gamma=\partial \Omega$ ) to get

$$
\begin{aligned}
\|\kappa\|_{k, \alpha, \Omega_{r_{0}}} & \leq C_{6}\left[\|J \kappa\|_{k-2, \alpha, \Omega_{r_{0} / 2}}+\|\kappa\|_{k, \alpha, \partial \Omega}+\|\kappa\|_{0, \Omega_{r_{0} / 2}}\right] \\
& \leq C_{7}\left[\|J \kappa\|_{k-2, \alpha ; \nu-2}+\|\kappa\|_{k, \alpha, \partial \Omega}+\|\kappa\|_{k-2,0 ; \nu}\right]
\end{aligned}
$$

Summing up (2.1.8) and (2.1.9), we get the desired estimate.

Later we will use the following corollary to Theorem 2.1 with $k=2$.

2.2. Corollary. If $\kappa \in C_{\mathrm{loc}}^{2, \alpha}\left(\Omega_{0}, T_{u} N\right) \cap C^{0,0 ; \nu}\left(\Omega_{0}, T_{u} N\right)$ is solution of $J_{u} \kappa=f$ with $\kappa \mid \partial \Omega \in C^{2, \alpha}(\partial \Omega, N)$ and $f \in C^{0, \alpha, \nu-2}\left(\Omega_{0}, T_{u} N\right)$, then $\kappa$ is a Jacobi field in $C^{2, \alpha ; \nu}\left(\Omega_{0}, T_{u} N\right)$.

Proof : This is implied by the estimates (2.1.1) in Theorem 2.1:

$$
\|\kappa\|_{2, \alpha ; \nu} \leq C\left[\|\kappa\|_{0,0 ; \nu}+\|\kappa\|_{2, \alpha, \partial \Omega}+\|f\|_{0, \alpha, \nu-2}\right]
$$

In order to apply Corollary 2.2, we need that $\kappa \in C^{0,0 ; \nu}\left(\Omega_{0}, T_{u} N\right)$, or, $|\kappa(x)| \leq C \rho^{\nu}(x)$ for some constant $C$. This may not true for a solution $\kappa \in C_{\mathrm{loc}}^{2, \alpha}\left(\Omega_{0}, T_{u} N\right)$ of $J_{u} \kappa=f$. On the other hand we have the following estimate.

2.3. Theorem. Let $\mu \in \mathbf{R}, 0<\alpha<1$ and $f \in C^{0, \alpha, \mu}\left(\Omega_{0}, T_{u} N\right)$. If $\kappa \in C_{\mathrm{loc}}^{2, \alpha}\left(\Omega_{0}, T_{u} N\right) \cap L^{p}\left(\Omega, T_{u} N\right), p>1$ and $J_{u} \kappa=f$, then there is a constant $C$ such that for $x \in \Omega_{0}$,

$$
|\kappa(x)| \leq C\left[\rho(x)^{-m / p}\|\kappa\|_{L^{p}\left(B_{\rho(x) / 2}(x)\right)}+\rho(x)^{\mu+2}\|f\|_{0, \alpha, \mu}\right],
$$

where $\rho(x)=\operatorname{dist}(x, Z)$.

Proof : For $x \in \Omega_{0}$, let $R=\rho(x) / 4$ and $v \in C_{0}^{1}\left(\mathbf{B}_{R}(x)\right), v \geq 0$. Then as shown in [ML1, Lemma], we have,

$$
\int_{\mathbf{B}_{R}(x)} \nabla|\kappa| \cdot \nabla v \leq \int_{\mathbf{B}_{R}(x)} \frac{\Delta \kappa \cdot \kappa}{|\kappa|} v .
$$

Now since $J_{u} \kappa=0$, by (1.6.2) and the fact that $A(u) \perp T_{u} N$, we have

$$
\begin{aligned}
|\Delta \kappa \cdot \kappa| & \leq|D A(u)(\kappa, \nabla u, \nabla u) \cdot \kappa|+|f||\kappa| \\
& \leq C_{1}\left[|\nabla u|^{2}|\kappa|^{2}+|f||\kappa|\right]
\end{aligned}
$$


for some constant $C$ depending only on $N$. Now (2.3.2) and (2.3.3) imply that $|\kappa|$ is a subsolution of

$$
\Delta w+C_{1}|\nabla u|^{2} w\left|+C_{1}\right| f \mid=0
$$

in the sense that

$$
\int_{\mathbf{B}_{R}(x)} \nabla|\kappa| \nabla v-C_{1}|\nabla u|^{2}|\kappa| v-C_{1}|f| v \leq 0
$$

for $v \in C_{0}^{1}\left(\mathbf{B}_{R}(x)\right), v \geq 0$. By Theorem 8.17 in $[\mathbf{G T}]$, for $p>1$ and $q>m$, there exists a constant $C_{3}$ depending only on $m, p$ and $q$ such that

$$
\sup _{\mathbf{B}_{R}(x)}|\kappa| \leq C\left[R^{-m / p}\|\kappa\|_{L^{p}\left(\mathbf{B}_{2 R}(x)\right)}+R^{2(1-m / q)}\|f\|_{L^{q / 2}\left(\mathbf{B}_{2 R}(x)\right)}\right] .
$$

Note that

$$
\|f\|_{L^{q / 2}\left(\mathbf{B}_{2 R}(x)\right)} \leq \rho(x)^{\mu+2 m / q}\|f\|_{0, \alpha, \mu} .
$$

So (2.3.7) and (2.3.6) imply (2.3.1).

\section{$\S$ 3. Strict Minimality and Stability Under Perturbations}

The main results of this section are that stability and minimality of harmonic maps are preserved under small perturbations. Here the singular sets can be as general as in Section 1. For the stability of perturbed harmonic maps with isolated singularities, a similar result was proved in [ML1]. For related results on minimal surfaces, see $[\mathbf{H S}]$ and $[\mathbf{S N} \mathbf{1}]$.

As in section 1, let $\Omega \subset \mathbf{R}^{m}$ be bounded smooth (say $C^{3}$ ) domain and $N \subset \mathbf{R}^{p}$ is a smooth compact submanifold (say $C^{4}$ ), $m \geq 3, p \geq 3$. Let $Z$ be a fixed compact subset of $\Omega$ having finite $m-3$ dimensional Minkowski content $\mathcal{M}^{m-3}(Z)<\infty[\mathbf{F}, 3.2 .37]$ and $\Omega_{0}=\Omega \backslash Z$. We consider the maps in the space $C^{1 ; 0}\left(\Omega_{0}, N\right)$ that is defined by

$$
C^{1 ; 0}\left(\Omega_{0}, N\right)=\left\{u \in C_{\mathrm{loc}}^{1}\left(\Omega_{0}, N\right):\|u\|_{1 ; 0}<\infty\right\},
$$

where $\|u\|_{1 ; 0}=\sup _{x \in \Omega_{0}}[|u(x)|+\rho(x)|\nabla u(x)|]$. Note that $C^{1 ; 0}\left(\Omega_{0}, N\right)=C^{1,0 ; 0}\left(\Omega_{0}, N\right)$, defined as in Section 1 with $k=1, \alpha=0$ and $\nu=0$. Lemma 1.3 implies that

$$
C^{1 ; 0}\left(\Omega_{0}, N\right) \subseteq H^{1 ; 0}(\Omega, N) \subseteq H^{1}(\Omega, N) .
$$

Thus every map $u \in C^{1 ; 0}\left(\Omega_{0}, N\right)$ has finite energy $E(u)=\int_{\Omega}|\nabla u|^{2}$.

3.1. Definitions. For $u \in H^{1}(\Omega, N)$, let

$$
\begin{aligned}
& H_{u}^{1}(\Omega, N)=\left\{v \in H^{1}(\Omega, N): v=u \text { on } \partial \Omega\right\}, \\
& H_{0}^{1}\left(\Omega, T_{u} N\right)=\left\{\xi \in H_{0}^{1}\left(\Omega, \mathbf{R}^{p}\right): \xi \in T_{u} N\right\} .
\end{aligned}
$$

We say that $u \in C^{1 ; 0}\left(\Omega_{0}, N\right)$ is strictly minimizing (with rate $\mu$ ) if

$$
E(v)-E(u) \geq \mu \int_{\Omega} \frac{|v-u|^{2}}{\rho^{2}}, \quad \rho(x)=\operatorname{dist}(x, Z),
$$

for and some $\mu>0$ and all $v \in H_{u}^{1}(\Omega, N)$. If $\mu=0$, then $u$ is minimizing. 
We say that $u$ is strictly stable (with rate $\mu$ ) if

$$
\left.\frac{d^{2}}{d t^{2}}\right|_{t=0} E\left(u_{t}\right) \geq 2 \mu \int_{\Omega} \frac{|\xi|^{2}}{\rho^{2}}
$$

for some $\mu>0$ and all $\xi \in H_{0}^{1}\left(\Omega, T_{u} N\right) \cap L^{\infty}$, where $u_{t}:(-\varepsilon, \varepsilon) \rightarrow H^{1}(\Omega, N)$ is a curve satisfying $u_{0}=u$ and $\left.\frac{d}{d t}\right|_{t=0} u_{t}=\xi$. We say $u$ is stable if $\mu=0$.

Using Jacobi operator $J_{u}$ (define in 1.9), we can rewrite (3.1.3) as

$$
-\int_{\Omega} J_{u} \xi \cdot \xi \geq 2 \mu \int_{\Omega} \frac{|\xi|^{2}}{\rho^{2}}
$$

If (3.1.2) holds for $v \in H_{u}^{1}(\Omega, N)$ satisfying $\sup |v-u| \leq \delta$ for some $\delta>0$, we say that $u$ is strictly nearby minimizing, or strictly $\delta$-nearby minimizing with rate $\mu$.

We now give a formula being used to compare the energies of two maps. It was first shown by Macintosh and Simon $[\mathbf{M S}]$ for the case $\Omega_{0}=\mathbf{B}^{m} \backslash\{0\}$.

3.2. Theorem. Suppose that $u \in C^{1 ; 0}\left(\Omega_{0}, N\right)$ is a harmonic map and $v \in H_{u}^{1}(\Omega, N)$. Let $\xi=v-u$. Then

$$
E(v)-E(u)=\int_{\Omega}|\nabla \xi|^{2}-S_{u}(x, \xi),
$$

where

$$
S_{u}(x, q)=Q_{u}(x, q, q)+R_{u}(x, q)
$$

and $Q_{u}: \Omega_{0} \times \mathbf{R}^{p} \times \mathbf{R}^{p} \rightarrow \mathbf{R}$ is a bilinear form for each $x \in \Omega_{0}$ and $R_{u}: \Omega_{0} \times \mathbf{R}^{p} \rightarrow \mathbf{R}$ is a map that satisfies

$$
\left|R_{u}(x, q)\right|+\rho(x)\left|D_{x} R_{u}(x, q)\right|+|q|\left|D_{q} R_{u}(x, q)\right| \leq C_{0} \frac{|q|^{3}}{\rho^{2}}
$$

for some constant $C_{0}\left(N,\|u\|_{1,0}, n, p\right)$, all $q \in \mathbf{R}^{p}$ and $x \in \Omega_{0}$ with $u(x)+q \in N$.

Proof : For $v \in H_{u}^{1}(\Omega, N)$, let $\xi=v-u$. We have, by integration by parts and (1.4.2)

$$
\begin{aligned}
& \int_{\Omega}|\nabla v|^{2}-|\nabla u|^{2}=\int_{\Omega}|\nabla \xi|^{2}+2 \nabla \xi \cdot \nabla u \\
& =\int_{\Omega}|\nabla \xi|^{2}-2 \xi \cdot \Delta u=\int_{\Omega}|\nabla \xi|^{2}-2 \xi \cdot A(u)(\nabla u, \nabla u) .
\end{aligned}
$$

For $y \in N$ and $\xi \in \mathbf{R}^{p}$, let $\xi_{y}^{\top}$ and $\xi_{y}^{\perp}$ be the orthogonal projections of $\xi$ into $T_{y} N$ and $\left(T_{y} N\right)^{\perp}$, respectively. Define $B: N \times \mathbf{R}^{p} \rightarrow \mathbf{R}^{p}$ by

$$
B(y, \xi)=\xi_{y}^{\perp}-\frac{1}{2} A(y)\left(\xi_{y}^{\top}, \xi_{y}^{\top}\right) .
$$

Then there is a constant $C_{1}(N)$ such that for $y+\xi \in N$,

$$
|B(y, \xi)|+\left|D_{y} B(y, \xi)\right|+|\xi|\left|D_{\xi} B(y, \xi)\right| \leq C_{1}|\xi|^{3} .
$$

This holds because, locally, $N$ is the graph of second fundamental form $A$ over $T_{y} N$; that is, for $y+\xi \in N$,

$$
y+\xi=y+\xi_{y}^{\top}+\frac{1}{2} A(y)\left(\xi_{y}^{\top}, \xi_{y}^{\top}\right)+O\left(|\xi|^{3}\right) .
$$

For $\xi, \eta \in \mathbf{R}^{p}$ and $x \in \Omega_{0}$, define

$$
\begin{aligned}
& Q_{u}(x, \xi, \eta)=A(u(x))\left(\xi_{u(x)}^{\top}, \eta_{u(x)}^{\top}\right) \cdot A(u(x))(\nabla u(x), \nabla u(x)) . \\
& R_{u}(x, \xi)=2 B(u(x), \xi) \cdot A(u(x))(\nabla u(x), \nabla u(x)) .
\end{aligned}
$$

By the definition (3.2.2) of $S_{u},(3.2 .5),(3.2 .8)-(3.2 .9)$ and the fact that $A(u) \perp T_{u} N$, we verify

$$
S_{u}(x, \xi)=Q_{u}(x, \xi, \xi)+R_{u}(x, \xi)=2 \xi^{\perp} \cdot A(u)(\nabla u, \nabla u)=2 \xi \cdot A(u)(\nabla u, \nabla u) .
$$

This combined with (3.2.4) gives (3.2.1). The (3.2.3) follows from the definition (3.2.9) of $R_{u},(3.2 .6)$ and the fact $|\nabla u| \leq\|u\|_{1 ; 0} / \rho$. 
3.3. Remark. For $\xi=H_{0}^{1}\left(\Omega, T_{u} N\right)$, if we let $v=u_{t}:(\varepsilon, \varepsilon) \rightarrow H^{1}(\Omega, N)$ be a curve with $u_{0}=u$ and $\left.\frac{d}{d t}\right|_{t=0} u_{t}=\xi$ in (3.2.1), then (3.2.3) implies that

$$
\left.\frac{d^{2}}{d t^{2}}\right|_{t=0} E\left(u_{t}\right)=2 \int_{\Omega}|\nabla \xi|^{2}-Q_{u}(x, \xi, \xi) .
$$

In particular, if $N=\mathbf{S}^{p-1}$, we can take $R_{u}(x, \xi)=0$ and $S_{u}(x, \xi)=Q_{u}(x, \xi, \xi)=|\xi|^{2}|\nabla u|^{2}$; therefore, we have for $\xi=v-u$,

$$
E(v)-E(u)=\int_{\mathbf{B}^{n}}|\nabla \xi|^{2}-|\nabla u|^{2}|\xi|^{2} .
$$

Indeed, in this case, because $2 \xi \cdot u+|\xi|^{2}=0, A(u)(\xi, \eta)=-(\xi \cdot \eta) u$, we have, in (3.2.4)

$$
2 \xi \cdot A(u)(\nabla u, \nabla u)=-2 \xi \cdot u|\nabla u|^{2}=|\xi|^{2}|\nabla u|^{2}
$$

(If we apply theorem 3.2 , we will have $B(u, \xi)=-(\xi \cdot u)^{2} u / 2, Q_{u}(x, \xi, \xi)=|\nabla u|^{2}\left(|\xi|^{2}-(\xi \cdot u)^{2}\right.$ ) and $R_{u}(x, \xi)=|\nabla u|^{2}(\xi \cdot u)^{2}$.)

Thus in the case $N=\mathbf{S}^{p-1}$, both (3.1.2) and (3.1.3) can be written as

$$
\int_{\mathbf{B}^{n}}|\nabla \xi|^{2}-|\nabla u|^{2}|\xi|^{2} \geq \mu \int_{\Omega} \frac{|\xi|^{2}}{\rho^{2}}
$$

for all $\xi \in H_{0}^{1}\left(\Omega, \mathbf{R}^{p}\right)$ that satisfy $|\xi+u|=1$ and $\xi \in H_{0}^{1}\left(\Omega, T_{u} N\right)$, respectively.

Similarly, for $N=\mathbf{S}_{a}^{m}=\left\{(u, z) \in \mathbf{R}^{m+1}:|u|^{2}+\frac{|z|^{2}}{a^{2}}=1\right\}$, with $a>0$, if $(u, 0) \in H^{1}(\Omega, N)$ is an equator map, then for $(\xi, z) \in H_{0}^{1}\left(\Omega, \mathbf{R}^{p}\right)(p=m+1)$ satisfying $(u+\xi, z) \in H^{1}(\Omega, N)$, we have

$$
E((u+\xi, z))-E((u, 0))=\int_{\mathbf{B}^{n}}|\nabla \xi|^{2}-|\nabla u|^{2}|\xi|^{2}+\int_{\mathbf{B}^{n}}|\nabla z|^{2}-\left|\nabla \frac{u}{a}\right|^{2}|z|^{2} .
$$

3.4. Theorem. A harmonic map $u \in H^{1}(\Omega, N)$ is strictly stable if and only if $u$ is strictly nearby minimizing.

Proof : Suppose that $u$ is strictly minimizing with rate $\mu>0$. If $\xi \in H_{0}^{1}\left(\Omega, T_{u} N\right) \cap L^{\infty}$, then $u_{t}=\pi(u+t \xi)$ satisfies $\left.\frac{d}{d t}\right|_{t=0} u_{t}=\xi$ and $\left|u_{t}-u\right| \leq C|t||\xi|$. So when $t$ is sufficiently small, by strict nearby minimality of $u$,

$$
E\left(u_{t}\right)-E(u) \geq \mu \int_{\Omega} \frac{\left|u_{t}-u\right|^{2}}{\rho^{2}} .
$$

Thus

$$
\left.\frac{d^{2}}{d t^{2}}\right|_{t=0} E\left(u_{t}\right)=2 \lim _{t \rightarrow 0} \frac{E\left(u_{t}\right)-E(u)}{t^{2}} \geq 2 \mu \int_{\Omega} \frac{|\xi|^{2}}{\rho^{2}} .
$$

This shows the necessity.

To show sufficiency, suppose that $u \in C^{1 ; 0}\left(\Omega_{0}, N\right)$ is strictly stable with rate $\mu>0$. Let

$$
\xi \in H_{0}^{1}\left(\Omega, \mathbf{R}^{p}\right), \quad \xi+u \in N, \quad|\xi| \leq \delta,
$$

with $\delta>0$ being chosen.

Define $\eta=\xi_{u}^{\top}$ and $\chi=\xi_{u}^{\perp}=\xi-\eta$. Then $\eta \in H_{0}^{1}\left(\Omega, T_{u} N\right)$ and by strict stability of $u$ and formula

$$
\int_{\Omega}|\nabla \eta|^{2}-Q_{u}(x, \eta, \eta) \geq \mu \int_{\Omega} \frac{|\eta|^{2}}{\rho^{2}} .
$$


From the definition of $C^{1 ; 0}\left(\Omega_{0}, N\right)$ and the fact $N \in C^{4}$, we have

$$
\begin{gathered}
|\nabla u(x)| \leq \frac{\|u\|_{1 ; 0}}{\rho(x)}, \quad\|A\|_{C^{2}(N)} \leq C_{2} . \\
\left|\xi_{u}^{\top}\right| \leq|\xi|, \quad\left|\nabla \xi_{u}^{\top}\right| \leq C_{3}\left[|\nabla \xi|+\frac{|\xi|}{\rho}\right] .
\end{gathered}
$$

Here and in the following, unless otherwise indicated, the constants $C_{i}$ depend only on $N,\|u\|_{1 ; 0}, m$ and $p$. Now from (3.2.5), we have

$$
\chi=\xi_{u}^{\perp}=B(x, \xi)+\frac{1}{2} A(u)\left(\xi_{u}^{\top}, \xi_{u}^{\top}\right) .
$$

Using (3.2.6), (3.4.3) and (3.4.4), we get

$$
\begin{aligned}
|\chi| \leq C_{4}\left[|\xi|^{3}+\left|\xi_{u}^{\top}\right|^{2}\right] \leq 2 C_{4}|\xi|^{2} \leq 2 C_{4} \delta|\xi| . \\
|\nabla \chi| \leq\left|D_{u} B(u, \xi)\right||\nabla u|+\left|D_{\xi} B(u, \xi)\right||\nabla \xi|+ \\
+\frac{1}{2}\left|D_{u} A(u)\left(\nabla u, \xi_{u}^{\top}, \xi_{u}^{\top}\right)\right|+\left|A(u)\left(\nabla \xi_{u}^{\top}, \xi_{u}^{\top}\right)\right| \\
\leq C_{5}\left[\frac{|\xi|^{3}}{\rho}+|\nabla \xi||\xi|^{2}+\frac{\left|\xi_{u}^{\top}\right|^{2}}{\rho}+|\nabla \xi \| \xi|+\frac{|\xi|^{2}}{\rho}\right] \\
\leq C_{6} \delta\left[|\nabla \xi|+\frac{|\xi|}{\rho}\right] .
\end{aligned}
$$

By Schwartz's inequality, (3.4.5) and (3.4.6), we have the following

$$
\begin{aligned}
& |\nabla \chi|^{2} \leq 2 C_{6}^{2} \delta^{2}\left[|\nabla \xi|^{2}+\frac{|\xi|^{2}}{\rho^{2}}\right] \\
& \left(1-C_{7} \delta\right)|\xi|^{2} \leq|\eta|^{2} \leq\left(1+C_{7} \delta\right)|\xi|^{2} . \\
& |\nabla \eta|^{2} \leq\left(1+C_{8} \delta\right)|\nabla \xi|^{2}+C_{8} \delta \frac{|\xi|^{2}}{\rho^{2}} .
\end{aligned}
$$

Since $Q$ is bilinear and $\eta=\xi-\chi$,

$$
Q_{u}(x, \eta, \eta)=Q_{u}(x, \xi, \xi)-2 Q_{u}(x, \xi, \chi)+Q_{u}(x, \chi, \chi) .
$$

By definition of $Q,(3.4 .3)$ and (3.4.5),

$$
\begin{gathered}
\left|Q_{u}(x, \xi, \chi)\right|=\left|A(u)\left(\xi_{u}^{\top}, \chi_{u}^{\top}\right) \cdot A(u)(\nabla u, \nabla u)\right| \\
\leq C_{9}|\xi||\chi||\nabla u|^{2} \leq C_{10} \delta \frac{|\xi|^{2}}{\rho^{2}} . \\
\left|Q_{u}(x, \chi, \chi)\right|=\left|A(u)\left(\chi_{u}^{\top}, \chi_{u}^{\top}\right) \cdot A(u)(\nabla u, \nabla u)\right| \\
\leq C_{11}|\chi|^{2}|\nabla u|^{2} \leq C_{12} \delta^{2} \frac{|\xi|^{2}}{\rho^{2}} . \\
\left|Q_{u}(x, \xi, \xi)\right| \leq\left|A(u)\left(\xi_{u}^{\top}, \xi_{u}^{\top}\right) \cdot A(u)(\nabla u, \nabla u)\right| \leq C_{13} \frac{|\xi|^{2}}{\rho^{2}} .
\end{gathered}
$$

Thus (3.4.10)-(3.4.12) imply

$$
Q(x, \eta, \eta) \leq Q(x, \xi, \xi)+C_{14} \delta \frac{|\xi|^{2}}{\rho^{2}}
$$


and (3.4.13), (3.4.14) imply

$$
Q(x, \eta, \eta) \leq\left(1+C_{8} \delta\right) Q(x, \xi, \xi)+C_{15} \delta \frac{|\xi|^{2}}{\rho^{2}} .
$$

From (3.2.3),

$$
\left|R_{u}(x, \xi)\right| \leq C_{0} \delta \frac{|\xi|^{2}}{\rho^{2}}
$$

Putting (3.4.9), (3.4.15) and (3.4.8) into (3.4.2), we get

$$
\int_{\Omega}\left(1+C_{8} \delta\right)|\nabla \xi|^{2}+C_{8} \delta \frac{|\xi|^{2}}{\rho^{2}}-\left(1+C_{8} \delta\right) Q(x, \xi, \xi)+C_{15} \delta \frac{|\xi|^{2}}{\rho^{2}} \geq \int_{\Omega} \mu\left(1-C_{7} \delta\right) \frac{|\xi|^{2}}{\rho^{2}} .
$$

Combining this with the definition of $S_{u}(x, \xi)$ and (3.4.16), we get

$$
\left(1+C_{8} \delta\right)\left[\int_{\Omega}|\nabla \xi|^{2}-S_{u}(x, \xi)\right] \geq\left(\mu-C_{16} \delta\right) \int_{\Omega} \frac{|\xi|^{2}}{\rho^{2}} .
$$

Choose $\delta$ small so that $\mu_{1}=\left(\mu-C_{16} \delta\right) /\left(1+C_{8} \delta\right)>0$, then

$$
\int_{\Omega}|\nabla \xi|^{2}-S_{u}(x, \xi) \geq \mu_{1} \int_{\Omega} \frac{|\xi|^{2}}{\rho^{2}},
$$

for all $\xi$ satisfying (3.4.1). That is $u$ is strictly $\delta$-nearby minimizing with rate $\mu_{1}$.

3.5. Theorem. Suppose that $u \in C^{1 ; 0}\left(\Omega_{0}, N\right)$ is a strictly minimizing harmonic map. Then there is an $\varepsilon>0$ depending only on $\|u\|_{1 ; 0}, m$ and $p$, such that any harmonic map $v \in C^{1 ; 0}\left(\Omega_{0}, N\right)$ with $\|v-u\|_{1 ; 0} \leq \varepsilon$ is also strictly minimizing.

Proof : Take $\delta=\delta(N)>0$ such that for any

$$
x \in N_{4 \delta} \equiv\left\{x \in \mathbf{R}^{p}: \operatorname{dist}(x, N) \leq 4 \delta\right\},
$$

there exists unique $\pi(x) \in N$ such that $\operatorname{dist}(x, N)=|x-\pi(x)|$. Note that $\pi \in C^{3}$, since $N \in C^{4}$.

Suppose that $u \in C^{1 ; 0}\left(\Omega_{0}, N\right)$ is harmonic and strictly minimizing with rate $\mu>0$ and $v \in C^{1 ; 0}\left(\Omega_{0}, N\right)$ is harmonic satisfying

$$
\|v-u\|_{1 ; 0} \leq \varepsilon
$$

with $\varepsilon \in(0, \delta)$. We show that $v$ also strictly minimizing if $\varepsilon$ is small enough.

Suppose that $\eta \in H_{0}^{1}\left(\Omega, \mathbf{R}^{p}\right)$ and $\eta+v \in N$. Define $\xi=\pi(u+\eta)-u$, then $\xi$ is well defined because $\operatorname{dist}(u+\eta, N) \leq\|v-u\|_{1 ; 0} \leq \varepsilon \leq \delta$. Noticing that $\xi \in H_{0}^{1}\left(\Omega, \mathbf{R}^{p}\right)$ and $\xi+u \in N$, by strict minimality of $u$, we have

$$
\int_{\Omega}|\nabla \xi|^{2}-S_{u}(x, \xi) \geq \mu \int_{\Omega} \frac{|\xi|^{2}}{\rho^{2}} .
$$

Let $\chi=\xi-\eta=\pi(u+\eta)-(u+\eta)$. To estimate $\chi$ we consider the map

$$
\begin{aligned}
& F: Q_{2 \delta} \equiv\left\{(y, z) \in N_{2 \delta} \times \mathbf{R}^{p}, y+z \in N_{2 \delta}\right\} \rightarrow \mathbf{R}^{p} \\
& F(y, z)=\pi(y+z)-(y+z) .
\end{aligned}
$$


Since $\operatorname{dist}\left(\partial Q_{2 \delta}, Q_{\delta}\right) \geq \delta$ and $\pi \in C^{3}$, we can extend $F$ to $\mathbf{R}^{p} \times \mathbf{R}^{p} \rightarrow \mathbf{R}^{p}$ so that for some constant $C(\delta, N)$

$$
\left|D_{y}^{\alpha} D_{z}^{\beta} F\right| \leq C, \quad|\alpha|+|\beta| \leq 3 .
$$

Now since $F(v, \eta)=0$, we have

$$
F(u, \eta)=\int_{0}^{1} D_{y} F(v+t(u-v), \eta)(u-v) d t
$$

Using the fact that $F(u, 0)=0$ in this equality, we get

$$
\begin{aligned}
F(u, \eta) & =\int_{0}^{1}\left[D_{y} F(v+t(u-v), \eta)-D_{y} F(v+t(u-v), 0)\right](u-v) d t \\
& =\int_{0}^{1} \int_{0}^{1} D_{y} D_{z} F(v+t(u-v), s \eta)[(u-v) \otimes \eta] d t d s .
\end{aligned}
$$

From (3.5.4), (3.5.1) and by using Schwartz's inequality,

$$
\begin{gathered}
|\chi|=|F(u, \eta)| \leq\left|D_{y} D_{z} F\right||u-v \| \eta| \leq C_{2} \varepsilon|\eta| . \\
\left(1-C_{3} \delta\right)|\eta|^{2} \leq|\xi|^{2} \leq\left(1+C_{3} \delta\right)|\eta|^{2} . \\
|\nabla \chi| \leq C_{4}[|\nabla v|+|\nabla u|+|\eta|]|u-v||\eta|+ \\
+C_{4}[|\nabla u-\nabla v||\eta|+|u-v||\nabla \eta|] \\
\leq C_{5} \varepsilon\left[|\nabla \eta|+\frac{|\eta|}{\rho}\right] . \\
|\nabla \chi|^{2} \leq 2 C_{5}^{2} \varepsilon^{2}\left[\frac{|\eta|^{2}}{\rho^{2}}+|\nabla \eta|^{2}\right] . \\
|\nabla \xi|^{2} \leq\left(1+C_{6} \varepsilon\right)|\nabla \eta|^{2}+C_{6} \varepsilon \frac{|\eta|^{2}}{\rho^{2}} .
\end{gathered}
$$

Next we need to consider the maps $u_{t}=\pi(u+t(v-u)), t \in[0,1]$. As in (3.4.3) and (3.4.4) we have

$$
\begin{gathered}
\left|\nabla u_{t}(x)\right| \leq C_{7} \frac{\|u\|_{1 ; 0}+\delta}{\rho(x)}, \quad\|A\|_{C^{2}(N)} \leq C_{7}, \quad\|\pi\|_{C^{3}\left(N_{2 \delta}\right)} \leq C_{7}, \\
\left|\eta_{u_{t}}^{\top}\right| \leq|\eta|, \quad\left|\nabla \eta_{u_{t}}^{\top}\right| \leq C_{8}\left[|\nabla \eta|+\frac{|\eta|}{\rho}\right] .
\end{gathered}
$$

By definition (3.2.2) of $S_{u}$ and the relation $\xi=\eta+\chi$,

$$
\begin{aligned}
S_{u}(x, \xi)= & Q_{u}(x, \xi, \xi)+R_{u}(x, \xi) \\
= & Q_{u}(x, \eta, \eta)+2 Q_{u}(x, \eta, \chi)+Q_{u}(x, \chi, \chi)+R_{u}(x, \xi) \\
= & S_{v}(x, \eta)+\left[Q_{u}(x, \eta, \eta)-Q_{v}(x, \eta, \eta)\right]+ \\
& {\left[R_{u}(x, \xi)-R_{v}(x, \eta)\right]+2 Q_{u}(x, \eta, \chi)+Q_{u}(x, \chi, \chi) . }
\end{aligned}
$$

By definition (3.2.8) of $Q,(3.5 .10)$ and (3.5.11), we get

$$
\begin{aligned}
\left|Q_{u}(x, \eta, \eta)-Q_{v}(x, \eta, \eta)\right| & =\left|A\left(u_{t}\right)\left(\eta_{u_{t}}^{\top}, \eta_{u_{t}}^{\top}\right) \cdot A\left(u_{t}\right)\left(\nabla u_{t}, \nabla u_{t}\right)\right|_{t=0}^{t=1} \mid \\
& =\left|\int_{0}^{1} \frac{d}{d t}\left[A\left(u_{t}\right)\left(\eta_{u_{t}}^{\top}, \eta_{u_{t}}^{\top}\right) \cdot A\left(u_{t}\right)\left(\nabla u_{t}, \nabla u_{t}\right)\right] d t\right| \\
& \leq C_{9} \varepsilon \frac{|\eta|^{2}}{\rho^{2}} .
\end{aligned}
$$


Similarly, by definition $(3.2,9)$ of $R,(3.5 .10)-(3.5 .11)$ and (3.5.5)-(3.5.6),

$$
\begin{aligned}
\left|R_{u}(x, \xi)-R_{v}(x, \eta)\right| & \leq\left|R_{u}(x, \xi)-R_{v}(x, \xi)\right|+\left|R_{v}(x, \xi)-R_{v}(x, \eta)\right| \\
& \leq C_{10}|v-u| \frac{|\eta|^{2}}{\rho^{2}}+C_{10}|\eta-\xi| \frac{|\eta|^{2}+|\xi|^{2}}{\rho^{2}} \leq C_{11} \varepsilon \frac{|\eta|^{2}}{\rho^{2}} .
\end{aligned}
$$

By definition (3.2.8) of $Q$ and (3.5.5) (similar to (3.4.11)-(3.4.12)),

$$
\begin{gathered}
\left|Q_{u}(x, \eta, \chi)\right| \leq C_{12}|\eta||\chi||\nabla u|^{2} \leq C_{13} \varepsilon \frac{|\eta|^{2}}{\rho^{2}} . \\
\left|Q_{u}(x, \chi, \chi)\right| \leq{ }_{14} C \varepsilon^{2} \frac{|\eta|^{2}}{\rho^{2}} .
\end{gathered}
$$

Putting (3.5.13)-(3.5.16) into (3.5.12), we get

$$
-S_{u}(x, \xi) \leq-S_{v}(x, \eta)+C_{15} \varepsilon \frac{|\eta|^{2}}{\rho^{2}} .
$$

To write (3.5.17) into the needed form, we note that

$$
\left|S_{v}(x, \eta)\right| \leq\left|Q_{v}(x, \eta, \eta)\right|+\left|R_{v}(x, \eta)\right| \leq C_{16} \frac{|\eta|^{2}}{\rho^{2}},
$$

thus (3.5.17) together with (3.5.18) gives

$$
-S_{u}(x, \xi) \leq-\left(1+C_{6} \varepsilon\right) S_{v}(x, \eta)+C_{17} \varepsilon \frac{|\eta|^{2}}{\rho^{2}}
$$

Substituting (3.5.9), (3.5.19) and (3.5.6) into (3.5.2), we get

$$
\left(1+C_{6} \varepsilon\right) \int_{\Omega}|\nabla \eta|^{2}-S_{v}(x, \eta) \geq \mu\left(1-C_{18} \varepsilon\right) \int_{\Omega} \frac{|\eta|^{2}}{\rho^{2}} .
$$

Choose $\varepsilon$ small so that $\left.\mu_{1}=\left(\mu-C_{18} \varepsilon\right)\right) /\left(1+C_{6} \varepsilon\right)>0$, then

$$
\int_{\Omega}|\nabla \eta|^{2}-S_{v}(x, \eta) \geq \mu_{1} \int_{\Omega} \frac{|\eta|^{2}}{\rho^{2}}
$$

for all $\eta \in H_{0}^{1}\left(\Omega, \mathbf{R}^{p}\right)$ satisfying $\eta+v \in N$. This shows that $v$ is strictly minimizing with rate $\mu_{1}$, if $\|v-u\|_{1 ; 0} \leq \varepsilon$.

Remark 3.6. From the proof of Theorem 3.5, we see that $\varepsilon$ and $\mu_{1}$ depend only on $\|u\|_{1 ; 0}, \mu, m$ and $p$; furthermore we have $\mu_{1} \rightarrow \mu$ when $\varepsilon \rightarrow 0+$. Thus we have the following

Corollary 3.7. If $u_{i} \in C^{1 ; 0}\left(\Omega_{0}, N\right)$ is a sequence of strictly minimizing harmonic maps with rates $\mu_{i} \geq \mu>0$ and $u_{i} \rightarrow u$ in $C^{1 ; 0}\left(\Omega_{0}, N\right)$, then $u$ is also a strictly minimizing harmonic map with rate $\mu$.

Proof : Since $u_{i} \rightarrow u$ in $C^{1 ; 0}\left(\Omega_{0}, N\right)$, we have $u_{i} \rightarrow u$ in $H^{1}(\Omega, N)$ and $\left\|u_{i}\right\|_{1 ; 0} \leq K$ for some $K \in(0, \infty)$. So $u$ is harmonic. By Remark 3.6, there are an $\varepsilon=\varepsilon(K, \mu, m, p)>0$ and $\mu_{1}=\mu_{1}(K, \mu, m, p)>0$ such that if $v \in C^{1 ; 0}\left(\Omega_{0}, N\right)$ is harmonic and $\left\|u_{i}-v\right\|_{1 ; 0} \leq \varepsilon$ for some $i$, then $v$ is strictly minimizing with rate $\mu_{1}$. In particular, when $i$ large, we have $\left\|u_{i}-u\right\|_{1 ; 0} \leq \varepsilon$; so $u$ is strictly minimizing with rate $\mu_{1}$. Note that $\mu_{1}$ can be arbitrarily close to $\mu$ if $\varepsilon$ is close to 0 . Therefore $u$ is actually strictly minimizing with rate $\mu$. 
By the same technique in Theorem 3.5, we can show that strict stability and strict nearby minimality are also preserved under small perturbation. We have the following theorem, whose proof is omitted.

3.8. Theorem. If $u \in C^{1 ; 0}\left(\Omega_{0}, N\right)$ is strictly minimizing (strictly stable, or strictly nearby minimizing) harmonic map with rate $\mu>0$, then there are positive constants $\varepsilon$ and $\mu_{1}$ depending on $\|u\|_{1 ; 0}, \mu, N, m, p$ such that any harmonic map $v \in C^{1 ; 0}\left(\Omega_{0}, N\right)$ with $\|v-u\|_{1 ; 0} \leq \varepsilon$ is strictly minimizing (strictly stable, or strictly nearby minimizing) with rate $\mu_{1}$.

If $u_{i} \in C^{1 ; 0}\left(\Omega_{0}, N\right)$ is a sequence of strictly minimizing (strictly stable, or strictly nearby minimizing) harmonic maps with rates $\mu_{i} \geq \mu>0$ and $u_{i} \rightarrow u$ in $C^{1 ; 0}\left(\Omega_{0}, N\right)$, then $u$ is a also strictly minimizing (strictly stable, or strictly nearby minimizing) harmonic map with rate $\mu$.

\section{$\S$ 4. Structure of the Space of Harmonic Maps}

4.1. Here we study the structure of the space of harmonic maps in $C^{k, \alpha ; 0}\left(\mathbf{B}_{1}^{m} \backslash\{0\}, N\right)$ near a homogeneous harmonic map that is singular only at the origin. We mainly prove that this space is Banach manifold (Theorem 4.8) and give precise estimates on the Fredholm indices of the Jacobi operator (Theorem 4.4). N. Smale has recently proved the Fredholm property of "conic" operators that include the Jacobi operator of a harmonic map with isolated singularities and unique tangent maps [SN3].

Let $\mathbf{B}=\mathbf{B}_{1}^{m}(0)$ and $Z=\{0\}$ and denote $\mathbf{B}_{0}=\mathbf{B}_{1}^{m}(0) \backslash\{0\}$. Suppose that $u: \mathbf{B}_{0} \rightarrow N$ is a homogeneous harmonic with $\varphi=u \mid \partial \mathbf{B} \in C^{2, \alpha}\left(\mathbf{S}^{m-1}, N\right)$. Then we have that $u(x)=\varphi\left(\frac{x}{|x|}\right)$ and $\varphi$ is also harmonic. Let $J_{\varphi}$ and $J_{u}$ be the Jacobi operators with respect to $\varphi$ and $u$ repectively. Using the polar coordinates $x=r \theta$ $\left(r=|x|, \theta \in \mathbf{S}^{m-1}\right)$ on $\mathbf{B}$, we can write, by (1.7.2),

$$
J_{\varphi} \eta=\Delta_{\mathbf{S}} \eta-2 A(\varphi)\left(\nabla_{\mathbf{S}} \varphi, \nabla_{\mathbf{S}} \eta\right)-D A(\varphi)\left(\eta, \nabla_{\mathbf{S}} \varphi, \nabla_{\mathbf{S}} \varphi\right)
$$

and

$$
J_{u} \kappa=\frac{\partial^{2}}{\partial r^{2}} \kappa+\frac{m-1}{r} \frac{\partial}{\partial r} \kappa+\frac{1}{r^{2}} J_{\varphi} \kappa(r \cdot)
$$

for $\eta \in C^{2, \alpha}\left(\mathbf{S}^{m-1}, T_{\varphi} N\right)$ and $\kappa \in C^{2, \alpha ; \nu}\left(\mathbf{B}_{0}, T_{u} N\right)$, where $\nabla_{\mathbf{S}}$ is the covariant differentiation on $\mathbf{S}^{m-1}$. Let $\mu_{1} \leq \mu_{2} \leq \cdots$ be the eigenvalues of $J_{\varphi}$ and $\eta_{1}, \eta_{2}, \ldots$, be the corresponding orthonormal (with respect to $\left.\langle\cdot, \cdot\rangle_{L^{2}\left(\mathbf{S}^{m-1}\right)}\right)$ eigenmaps in $C^{2, \alpha}\left(\mathbf{S}^{m-1}, T_{\varphi} N\right)$.

The following lemma gives a relation between the first eigenvalue $\mu_{1}$ of $J_{\varphi}$ and the first eigenvalue $\lambda$ of $J_{u}$.

\subsection{Lemma.}

$$
\lambda \geq \frac{(m-2)^{2}}{4}+\mu_{1}
$$

Proof : Argue as in the proof of $[\mathbf{C H S}]$.

4.3. Now for the Jacobi operator $J_{u}: C^{2, \alpha ; \nu}\left(\mathbf{B}_{0}, T_{u} N\right) \rightarrow C^{0, \alpha ; \nu-2}\left(\mathbf{B}_{0}, T_{u} N\right)$, we want to solve $J_{u} \kappa=f$ for $\kappa \in C^{2, \alpha ; \nu}\left(\mathbf{B}_{0}, T_{u} N\right)$ with $f \in C^{0, \alpha ; \nu-2}\left(\mathbf{B}_{0}, T_{u} N\right)$. Writing

$$
\kappa(r \theta)=\sum_{i=1}^{\infty} \kappa_{i}(r) \eta_{i}(\theta), \quad f(r \theta)=\sum_{i=1}^{\infty} f_{i}(r) \eta_{i}(\theta)
$$


with $\kappa_{i}(r)=\left\langle\kappa(r \cdot), \eta_{i}\right\rangle_{L^{2}\left(\mathbf{S}^{m-1}\right)}, f_{i}(r)=\left\langle f(r \cdot), \eta_{i}\right\rangle_{L^{2}\left(\mathbf{S}^{m-1}\right)}$, then formally, $J_{u} \kappa=f$ is equivalent to

$$
\kappa_{i}^{\prime \prime}(r)+\frac{m-1}{r} \kappa_{i}^{\prime}-\frac{1}{r^{2}} \mu_{i}=f_{i}(r), \quad i=1,2,3, \ldots,
$$

which are nonhomogeneous Euler equations. Let for $i=1,2,3, \ldots$,

$$
\gamma_{i}=\frac{2-m}{2}+\sqrt{\frac{(m-2)^{2}}{4}+\mu_{i}}, \quad \gamma_{i}^{-}=\frac{2-m}{2}-\sqrt{\frac{(m-2)^{2}}{4}+\mu_{i}} .
$$

Then a general solution $g_{i}$ of the homogeneous equation associated with (4.3.2) is

$$
g_{i}(r)= \begin{cases}a_{i} \operatorname{Rer}^{\gamma_{i}}+b_{i} I m r^{\gamma_{i}}, & i \in I_{1} \equiv\left\{i: \mu_{i}<-\frac{(m-2)^{2}}{4}\right\} \\ a_{i} r^{\frac{2-m}{2}}+b_{i} r^{\frac{2-m}{2}} \log r, & i \in I_{2} \equiv\left\{i: \mu_{i}=-\frac{(m-2)^{2}}{4}\right\} \\ a_{i} r^{\gamma_{i}}+b_{i} r^{\gamma_{i}^{-}}, & i \in I_{3} \equiv\left\{i: \mu_{i}>-\frac{(m-2)^{2}}{4}\right\}\end{cases}
$$

with arbitrary constants $a_{i}$ and $b_{i}$. Note that $\gamma_{i}=\bar{\gamma}_{i}^{-}$are complex for $i \in I_{1}, \gamma_{i}=\gamma_{i}^{-}=\frac{2-m}{2}$ for $i \in I_{2}$ and $\gamma_{i}^{-}<\frac{2-m}{2}<\gamma_{i}$ for $i \in I_{3}$. A particular solution $F_{i}(r)$ of (4.3.2) is

$$
F_{i}(r)= \begin{cases}\operatorname{Re} r^{\gamma_{i}} \int_{0}^{r} \tau^{-m+1-2 \gamma_{i}} \int_{0}^{\tau} s^{m-1+\gamma_{i}} f_{i}(s) d s d \tau, & \gamma_{i}<\nu ; \\ \operatorname{Re} r^{\gamma_{i}} \int_{1}^{r} \tau^{-m+1-2 \gamma_{i}} \int_{0}^{\tau} s^{m-1+\gamma_{i}} f_{i}(s) d s d \tau, & \gamma_{i} \geq \nu .\end{cases}
$$

Note that $F_{i}(r)$ satisfies that $\left|F_{i}(r)\right| \leq C r^{\nu}$ for $i$ such that $\gamma_{i} \neq \nu$. Thus (4.3.2) has a general solution $\kappa_{i}(r)=g_{i}(r)+F_{i}(r)$.

Let

$$
\mathcal{G}(r \theta)=\sum_{i=1}^{\infty} g_{i} \eta_{i}(\theta), \quad \mathcal{F}(f)(r \theta)=\sum_{i=1}^{\infty} F_{i}(r) \eta_{i}(\theta),
$$

then the solution $\kappa$ of $J_{u} \kappa=f$ can be written

$$
\kappa(r \theta)=\mathcal{G}(r \theta)+\mathcal{F}(f)(r \theta) .
$$

Denote by $K_{\nu 0}\left(J_{u}\right)$ and $K_{\nu}\left(J_{u}\right)$ be the kernels of $J_{u}$ in $C_{0}^{2, \alpha ; \nu}\left(\mathbf{B}_{0}, T_{u} N\right)$ and $C^{2, \alpha ; \nu}\left(\mathbf{B}_{0}, T_{u} N\right)$, respectively, and $R_{\nu}\left(J_{u}\right)=J_{u}\left(C_{0}^{2, \alpha ; \nu}\left(\mathbf{B}_{0}, T_{u} N\right)\right)$.

\subsection{Theorem.}

(a). If $\nu \neq \gamma_{j}$ and $\nu>\frac{2-m}{2}$, then

$$
K_{\nu}\left(J_{u}\right)=\left\{\kappa \in C^{2, \alpha ; \nu}\left(\mathbf{B}_{0}, T_{u} N\right): \kappa(r \theta)=\sum_{\gamma_{i}>\nu} a_{i} r^{\gamma_{i}} \eta_{i}(\theta)\right\} .
$$

(b). If $\nu \neq \gamma_{j}$, then

$$
\operatorname{dim}\left(K_{\nu 0}\left(J_{u}\right)\right)= \begin{cases}\#\left\{i: \nu \leq \text { Re } \gamma_{i}^{-}\right\}, & \text {if } \nu<\frac{2-m}{2} \\ \#\left\{i: \gamma_{i} \text { is complex }\right\}, & \text { if } \nu=\frac{2-m}{2} \\ 0, & \text { if } \nu>\frac{2-m}{2}\end{cases}
$$

and

$$
\operatorname{codim}\left(R_{\nu}\left(J_{u}\right)\right)=\#\left\{i: \gamma_{i}<\nu\right\}
$$


Proof : Suppose $\kappa \in C^{2, \alpha ; \nu}\left(\mathbf{B}_{0}, T_{u} N\right)$ satisfies $J_{u} \kappa=0$, then $\kappa(r \theta)=\mathcal{G}(r \theta)$ with

$$
|\kappa(r \cdot)|_{L^{2}\left(\mathbf{S}^{m-1}\right)}=\sum_{i=1}^{\infty} g_{i}^{2}(r)
$$

where $g_{i}$ are given in (4.3.3). Since $\nu>\frac{2-m}{2}$,

$$
\kappa(r \theta)=\sum_{\gamma_{i}>\nu} a_{i} r^{\gamma_{i}} \eta_{i}(\theta)
$$

Conversely, any $\kappa \in C^{2, \alpha ; \nu}\left(\mathbf{B}_{0}, T_{u} N\right)$ of this form is in $K_{\nu}\left(J_{u}\right)$. This shows (a).

To show (b) when $\nu<\frac{2-m}{2}$, suppose that $J_{u} \kappa=0$ and $\kappa \in C_{0}^{2, \alpha ; \nu}\left(\mathbf{B}_{0}, T_{u} N\right)$, then from (4.4.1), we must have $\left|g_{i}(r)\right|=O\left(r^{\nu}\right)$ and $g_{i}(1)=0$. Thus $\kappa$ has the following expansion

$$
\kappa(r \theta)=\sum_{i \in I_{1}} b_{i} I m r^{\gamma_{i}}+\sum_{i \in I_{2}} b_{i} r^{\frac{2-m}{2}} \log r+\sum_{i \in I_{3} \cap\left\{\gamma_{i}^{-}>\nu\right\}} a_{i}\left(r^{\gamma_{i}}-r^{\gamma_{i}^{-}}\right) .
$$

Thus $\operatorname{dim}\left(K_{\nu}\left(J_{u}\right)\right)=\#\left\{i: \nu \leq R e \gamma_{i}^{-}\right\}$. The other cases $\nu \geq \frac{2-m}{2}$ are similar.

Now we estimate $\operatorname{codim}\left(R_{\nu}\left(J_{u}\right)\right)$. First we show that $\mathcal{F}(f)$ is a solution of $J_{u} \kappa=f$ in $C^{2, \alpha ; \nu}\left(\mathbf{B}_{0}, T_{u} N\right)$ for $f \in C^{0, \alpha ; \nu-2}\left(\mathbf{B}_{0}, T_{u} N\right)$. Write

$$
\mathcal{F}(f)=\sum_{i=1}^{\infty} F_{i}(r) \eta_{i}(\theta)=\sum_{\gamma_{i}<\nu} F_{i}(r) \eta_{i}(\theta)+\sum_{\gamma_{i}<\nu} F_{i}(r) \eta_{i}(\theta) \equiv \mathcal{F}_{1}(f)+\mathcal{F}_{2}(f)
$$

Clearly, being a finite sum, $\mathcal{F}_{1}(f) \in C^{2, \alpha ; \nu}\left(\mathbf{B}_{0}, T_{u} N\right)$.

For $\mathcal{F}_{2}(f) \in C^{2, \alpha ; \nu}\left(\mathbf{B}_{0}, T_{u} N\right)$, it is shown in [SN2] that

$$
\left|\mathcal{F}_{2}(f)(r \cdot)\right|_{L^{2}\left(\mathbf{S}^{m-1}\right)} \leq C_{2} r^{\nu}|f|_{0, \alpha ; \nu-2}
$$

This implies that $\left|\mathcal{F}_{2}(f)\right|_{L^{2}\left(\mathbf{B}_{r}\right)} \leq C_{2} r^{\nu+m / 2}$. By 2.3, we have that $\left|\mathcal{F}_{2}(f)\right| \leq C_{3} r^{\nu}$, or, $\mathcal{F}_{2}(f) \in$ $C^{0,0 ; \nu}\left(\mathbf{B}_{0}, T_{u} N\right)$. Corollary 2.2 then implies that $\mathcal{F}_{2}(f) \in C^{2, \alpha ; \nu}\left(\mathbf{B}_{0}, T_{u} N\right)$.

So $\mathcal{F}(f)$ is a solution of $J_{u} \kappa=f$ in $C^{2, \alpha ; \nu}\left(\mathbf{B}_{0}, T_{u} N\right)$. Since $\mathcal{F}(f)\left|\mathbf{S}^{m-1}=\mathcal{F}_{1}(f)\right| \mathbf{S}^{m-1}, u \in$ $C_{0}^{2, \alpha ; \nu}\left(\mathbf{B}_{0}, T_{u} N\right)$ iff $\mathcal{F}_{1}(f) \mid \mathbf{S}^{m-1}=0$, therefore,

$$
R_{\nu}\left(J_{u}\right)=\operatorname{kernel}\left(\mathcal{F}_{1} \mid \mathbf{S}^{m-1}\right)
$$

(4.4.3) implies that $\operatorname{codim}\left(R_{\nu}\left(J_{u}\right)\right)=\operatorname{rank}\left(\mathcal{F}_{1} \mid \mathbf{S}^{m-1}\right)=\#\left\{j: \gamma_{j}<\nu\right\}$.

In the rest of this section, we fix a $\nu \in\left(0, \gamma_{L}\right)$, where $\gamma_{L}=\min \left\{\gamma_{i}>0\right\}$. Denote $\mathcal{I}_{1}=\{1,2, \ldots, L-1\}$ and $\mathcal{I}_{2}=\{L, L+1, \ldots$,$\} . Define for j=1,2$,

$$
\begin{aligned}
& C_{j}^{2, \alpha ; \nu}\left(\mathbf{B}_{0}, T_{u} N\right)=\left\{\xi \in C^{2, \alpha ; \nu}\left(\mathbf{B}_{0}, T_{u} N\right): \xi(r \theta)=\sum_{i \in \mathcal{I}_{j}} a_{i}(r) \eta_{i}(\theta)\right\} \\
& C_{j}^{2, \alpha}\left(\mathbf{S}^{m-1}, T_{u} N\right)=\left\{\psi \in C^{2, \alpha}\left(\mathbf{S}^{m-1}, T_{u} N\right): \psi(\theta)=\sum_{i \in \mathcal{I}_{j}} a_{i} \eta_{i}(\theta)\right\}
\end{aligned}
$$

Denote

$$
C_{*}^{2, \alpha ; \nu}\left(\mathbf{B}_{0}, T_{u} N\right)=\left\{\kappa \in C^{2, \alpha ; \nu}\left(\mathbf{B}_{0}, T_{u} N\right): \kappa \mid \mathbf{S}^{m-1} \in C_{1}^{2, \alpha ; \nu}\left(\mathbf{B}_{0}, T_{u} N\right)\right\} .
$$

Let $\Pi_{j}$ be the projection from $C^{2, \alpha ; \nu}\left(\mathbf{B}_{0}, T_{u} N\right)$ to $C_{j}^{2, \alpha ; \nu}\left(\mathbf{B}_{0}, T_{u} N\right)$ and denote $\xi_{j}=\Pi_{j} \xi$. We have the following 
4.5. Corollary. For $\nu \in\left(0, \gamma_{L}\right), J_{u}$ is injective and $\operatorname{codim}\left(R_{\nu}\left(J_{u}\right)\right)=\operatorname{index}(\varphi)+\operatorname{nullity}(\varphi)$.

4.6. Theorem.

(a). For any $\psi \in C^{2, \alpha}\left(\mathbf{S}^{m-1}, T_{u} N\right)$ and $f \in C^{0, \alpha ; \nu-2}\left(\mathbf{B}_{0}, T_{u} N\right)$, there is a unique $\kappa \in C^{2, \alpha ; \nu}\left(\mathbf{B}_{0}, T_{u} N\right)$ such that

$$
\begin{cases}J_{u} \kappa=f, & \text { on } \mathbf{B} ; \\ \Pi_{2}(\kappa-\psi)=0, & \text { on } \mathbf{S}^{m-1}\end{cases}
$$

(b). $J_{u}: C_{*}^{2, \alpha ; \nu}\left(\mathbf{B}_{0}, T_{u} N\right) \rightarrow C^{0, \alpha ; \nu-2}\left(\mathbf{B}_{0}, T_{u} N\right)$ is an isomorphism.

Proof : We want to solve $J_{u} \kappa=f$ on $\mathbf{B}$ and $\kappa=\tau$ on $\mathbf{S}^{m-1}$ for some $\tau$ with $\Pi_{2}(\psi-\tau)=0$.

Let $\bar{\tau}$ be any extension of $\tau$ to $\mathbf{B}$ and $\xi=\kappa-\bar{\tau}$, then solving (4.6.1) is equivalent to solving the following

$$
J_{u} \xi=f-J_{u} \bar{\tau}, \text { on } \mathbf{B}, \quad \xi \mid \mathbf{S}^{m-1}=0, \text { on } \mathbf{S}^{m-1} .
$$

By (4.4.3), (4.6.2) is solvable if and only if $\mathcal{F}_{1}\left(f-J_{u} \bar{\tau}\right) \mid \mathbf{S}^{m-1}=0$.

By (4.3.4) and (4.4.2), both $\mathcal{F}_{1}\left(J_{u} \bar{\tau}\right)=\mathcal{F}_{1}\left(J_{u} \Pi_{1} \bar{\tau}\right)$ and $\Pi_{1} \bar{\tau}$ are solutions of $J_{u} \kappa=J_{u} \Pi_{1} \bar{\tau}$ on $\mathbf{B}$ in $C_{1}^{2, \alpha ; \nu}\left(\mathbf{B}_{0}, T_{u} N\right)$, therefore $\left.\mathcal{F}_{1}\left(J_{u} \bar{\tau}\right)-\Pi_{1} \bar{\tau} \in K_{\nu}\left(J_{u}\right) \subset C_{2}^{2, \alpha ; \nu}\left(\mathbf{B}_{0}, T_{u} N\right)\right)$. It follows that $\mathcal{F}_{1}\left(J_{u} \bar{\tau}\right)=\Pi_{1} \bar{\tau}$ and so $\mathcal{F}_{1}\left(J_{u} \bar{\tau}\right)\left|\mathbf{S}^{m-1}=\Pi_{1} \bar{\tau}\right| \mathbf{S}^{m-1}=\Pi_{1} \tau \mid \mathbf{S}^{m-1}$. Thus, if we take $\tau=\Pi_{2} \psi+\mathcal{F}_{1}(f) \mid \mathbf{S}^{m-1}$, then $\mathcal{F}_{1}\left(f-J_{u} \bar{\tau}\right) \mid \mathbf{S}^{m-1}=0$, so (4.6.2) has a unique solution $\xi$ and $\kappa=\xi+\bar{\tau}$ is therefore the needed solution of (4.6.1).

If $\kappa \in C_{*}^{2, \alpha ; \nu}\left(\mathbf{B}_{0}, T_{u} N\right)$ and $J_{u} \kappa=0$, then

$$
\kappa(r \theta)=\sum_{i \in \mathcal{I}_{2}} a_{i} r^{\gamma_{i}} \eta_{i}(\theta)
$$

and $\Pi_{2} \kappa \mid \mathbf{S}^{m-1}=\sum_{i \in \mathcal{I}_{2}} a_{i} \eta_{i}(\theta)=0$, which implies that $a_{i}=0$ for all $i \in \mathcal{I}_{2}$. So $J_{u}$ is injective.

If $f \in C^{0, \alpha ; \nu-2}\left(\mathbf{B}_{0}, T_{u} N\right)$, then by (a) for $\psi=0$ there is a unique solution $\kappa \in C^{2, \alpha ; \nu}\left(\mathbf{B}_{0}, T_{u} N\right)$ of $J_{u} \kappa=f$ with $\Pi_{2}(\kappa) \mid \mathbf{S}^{m-1}=0$. So $\kappa$ is a solution in $C_{*}^{2, \alpha ; \nu}\left(\mathbf{B}_{0}, T_{u} N\right)$, that is, $J_{u}$ is onto. This shows that $J_{u}$ is an isomorphism.

Now we describe the local structure of $\mathcal{H}$ near a homogeneous harmonic map.

4.7. Theorem. There exist a neighborhood $U$ of 0 in $C_{2}^{2, \alpha}\left(\mathbf{S}^{m-1}, T_{u} N\right)$, a neighborhood $V$ of $u$ in $C^{2, \alpha ; 0}\left(\mathbf{B}_{0}, N\right)$ and a smooth map $F: U \rightarrow V$ such that

(a). For any $\psi \in U, F(\psi)$ is a harmonic map. Furthermore, $D F(0)$ is an immersion.

(b). There is an $\epsilon>0$ such that any harmonic map $v \in V_{\epsilon}=\left\{v \in C^{2, \alpha ; 0}\left(\mathbf{B}_{0}, N\right):\|v-u\|_{2, \alpha ; \nu}<\epsilon\right\}$ is obtained in this way.

(c). $U$ and $\epsilon$ can be chosen so that $V_{\epsilon} \cap \mathcal{H}$ is a smooth manifold $C^{2}$ diffeomorphic to $U$.

The tangent space $T_{u} \mathcal{H}=K_{\nu}\left(J_{u}\right)$. In particular, all Jacobi fields are integrable.

Proof : Any $\psi \in C_{2}^{2, \alpha}\left(\mathbf{S}^{m-1}, T_{u} N\right)$ can be extended to a Jacobi field $\bar{\psi} \in K_{\nu}\left(J_{u}\right)$. That is, if $\psi(\theta)=\sum_{i \in \mathcal{I}_{2}} a_{i} \eta_{i}(\theta)$, then

$$
\bar{\psi}(r \theta)=\sum_{i \in \mathcal{I}_{2}} a_{i} r^{\gamma_{i}} \eta_{i}(\theta) .
$$

(See Theorem 4.4). Consider the map

$$
\Psi: C_{2}^{2, \alpha}\left(\mathbf{S}^{m-1}, T_{u} N\right) \times C_{*}^{2, \alpha ; \nu}\left(\mathbf{B}_{0}, T_{u} N\right) \rightarrow C^{0, \alpha ; \nu-2}\left(\mathbf{B}_{0}, T_{u} N\right)
$$


defined by

$$
\Psi(\psi, \kappa)=P_{u} \circ H(\pi(u+\bar{\psi}+\kappa)) .
$$

We note the following fact: if $\|v-u\|_{2, \alpha ; \nu}$ is small, then

$$
H(v)=0 \Longleftrightarrow P_{u} \circ H(v)=0 .
$$

Indeed, if $P_{u} \circ H(v)=0$, then

$$
\begin{aligned}
\langle H(v), H(v)\rangle_{L^{2}} & =\left\langle H(v), H(v)-P_{u} \circ H(v)\right\rangle_{L^{2}} \\
& =\langle H(v),[D \pi(v)-D \pi(u)] \circ H(v)\rangle_{L^{2}} \\
& \leq\|D \pi(v)-D \pi(u)\|\langle H(v), H(v)\rangle_{L^{2}} .
\end{aligned}
$$

So $\langle H(v), H(v)\rangle_{L^{2}}=0$ when $v$ is close to $u$ so that $\|D \pi(v)-D \pi(u)\|<1$.

Note that $D_{2} \Psi(0,0) \kappa=P_{u} \circ D H(u) \kappa=J_{u} \kappa$. By Theorem 4.6, $J_{u}$ is an isomorphism.

By the implicit function theorem, there are neighborhoods $U$ of 0 in $C_{2}^{2, \alpha}\left(\mathbf{S}^{m-1}, T_{u} N\right)$ and $W$ of 0 in $C_{*}^{2, \alpha ; \nu}\left(\mathbf{B}_{0}, T_{u} N\right)$ and a smooth map $Q: U \rightarrow W$ such that for any $\psi \in U, Q(\psi)$ is the unique solution of

$$
\Psi(\psi, Q(\psi))=P_{u} \circ H(\pi(u+\bar{\psi}+Q(\psi)))=0 .
$$

Let $F(\psi)=\pi(u+\bar{\psi}+Q(\psi))$. Then $F$ is a smooth map from $U$ into some neighborhood $V$ of $u$ in $C^{2, \alpha ; 0}\left(\mathbf{B}_{0}, N\right)$. By (4.7.4) and (4.7.3), $F(\psi)$ is harmonic.

To show that $D F(0)$ is an immersion, first note that for $\xi \in C_{2}^{2, \alpha}\left(\mathbf{S}^{m-1}, T_{u} N\right)$,

$$
\xi_{*} \equiv D F(0) \xi \in C^{2, \alpha ; \nu}\left(\mathbf{B}_{0}, T_{u} N\right)
$$

and $\Pi_{2}\left(\xi_{*}-\xi\right)=0$ on $\mathbf{S}^{m-1}$. Indeed this follows from the fact that $\xi_{*}=D F(0) \xi=\bar{\xi}+D Q(0) \xi$, where $D Q(0) \xi \in C_{*}^{2, \alpha ; \nu}\left(\mathbf{B}_{0}, T_{u} N\right)$.

For $\xi \in C^{2, \alpha ; \nu}\left(\mathbf{B}_{0}, T_{u} N\right)$, define $\xi_{*}=\left(\Pi_{2} \xi \mid \mathbf{S}^{m-1}\right)_{*}$ and $\Lambda \xi=\left(\Pi_{2} \xi \mid \mathbf{S}^{m-1}, \xi-\xi_{*}\right)$. Then one readily sees that

$$
C^{2, \alpha ; \nu}\left(\mathbf{B}_{0}, T_{u} N\right) \stackrel{\Lambda}{\longrightarrow} C_{2}^{2, \alpha}\left(\mathbf{S}^{m-1}, T_{u} N\right) \times C_{*}^{2, \alpha ; \nu}\left(\mathbf{B}_{0}, T_{u} N\right)
$$

is an isomorphism. Consider the following sequence

$$
\begin{aligned}
C_{2}^{2, \alpha}\left(\mathbf{S}^{m-1}, T_{u} N\right) & \stackrel{D F(0)}{\longrightarrow} C^{2, \alpha ; \nu}\left(\mathbf{B}_{0}, T_{u} N\right) \\
& \stackrel{\Lambda}{\longrightarrow} C_{2}^{2, \alpha}\left(\mathbf{S}^{m-1}, T_{u} N\right) \times C_{*}^{2, \alpha ; \nu}\left(\mathbf{B}_{0}, T_{u} N\right) \stackrel{P r_{1}}{\longrightarrow} C_{2}^{2, \alpha}\left(\mathbf{S}^{m-1}, T_{u} N\right)
\end{aligned}
$$

where $P r_{1}$ is the projection. It is easy to see that this gives an identity. So $D F(0)$ is an immersion [L, II, 2].

Now we show that if $\epsilon$ is small and $v \in V_{\epsilon}=\left\{v:\|v-u\|_{2, \alpha ; \nu}<\epsilon\right\}$ is a harmonic map, then $v=F(\psi)$ with $\psi=\Pi_{2} \pi^{-1} v \mid \mathbf{S}^{m-1}$. Since $\pi$ is a locally a diffeomorphism between $T_{u} N$ and $N$ (near $u \in N$ ), we have that for each $x \in \mathbf{B}_{0}$, there exists a unique $\lambda(x) \in T_{u(x)} N$ such that

$$
v(x)=\pi(u(x)+\lambda(x)),
$$

and $\lambda$, denoted by $\lambda=\pi^{-1} v$, smoothly depends on $v$. Let $\psi=\Pi_{2} \lambda$ and $\kappa=\lambda-\bar{\psi}$, where $\bar{\psi}$ is defined by (4.7.1). Then $\kappa \in C_{*}^{2, \alpha ; \nu}\left(\mathbf{B}_{0}, T_{u} N\right)$ and

$$
v=\pi(u+\bar{\psi}+\kappa)
$$

That $v$ is harmonic implies that

$$
\Psi(\psi, \kappa)=P_{u} \circ H(\pi(u+\bar{\psi}+\kappa))=0 .
$$


By the uniqueness of $Q$ for small $\psi$, we get $\kappa=Q(\psi)$ and then $v=F(\psi)$.

That $V_{\epsilon} \cap \mathcal{H}$ is a manifold follows since $D F(0)$ is an immersion [ $\mathbf{L}$, II,2]. To prove that $T_{u} \mathcal{H}=K_{\nu}\left(J_{u}\right)$ we need only to show that if $\kappa \in C^{2, \alpha ; \nu}\left(\mathbf{B}_{0}, T_{u} N\right)$ is a Jacobi field, then there is a one parameter family of harmonic maps $u_{t} \in \mathcal{H}$ with $u_{0}=u$ and $\left.\frac{d}{d t}\right|_{t=0} u_{t}=\kappa$. Indeed since $\kappa \mid \mathbf{S}^{m-1} \in C_{2}^{2, \alpha}\left(\mathbf{S}^{m-1}, T_{u} N\right)$ (by Theorem 4.4(a)), by (a), $u_{t}=F(t \kappa)$ is a family of harmonic maps (for all small $t$ ) and we have

$$
\left.\frac{d}{d t}\right|_{t=0} u_{t}=\left.\frac{d}{d t}\right|_{t=0} F(t \kappa)=\kappa+D Q(0) \kappa
$$

(note that $\bar{\kappa}=\kappa$ ). In fact $D Q(0) \kappa=0$ because $D Q(0) \kappa$ is a Jacobi field in $C_{*}^{2, \alpha ; \nu}\left(\mathbf{B}_{0}, T_{u} N\right)$ on which $J_{u}$ is one-to-one. So $\left.\frac{d}{d t}\right|_{t=0} u_{t}=\kappa$.

\section{$\S$ 5. Applications to Some Examples of Homogeneous Harmonic Maps}

In this section, we apply Theorem 4.7 and Theorem 3.8 to some well-known examples of harmonic maps. We first examine the stability and minimality of those maps, some of which are well-known, while some others are new.

5.1. Lemma. If $u \in H^{1}(\mathbf{B}, N)$ and either $u$ is a stationary harmonic map with $\frac{\partial u}{\partial \mathbf{n}} \mid \mathbf{S}^{m-1} \in L^{2}\left(\mathbf{S}^{m-1}\right)$, (this holds, e.g., if $u \in H^{2,2}(\mathbf{B}, N)$ ), or $u \in C_{\mathrm{loc}}^{1}\left(\mathbf{B}_{0}, N\right)$ is a harmonic map, then

$$
\int_{\mathbf{S}^{m-1}}\left|\nabla_{\tan } u\right|^{2}=\int_{\mathbf{S}^{m-1}}\left|\frac{\partial u}{\partial \mathbf{n}}\right|^{2}+(m-2) \int_{\mathbf{B}}|\nabla u|^{2} .
$$

Proof : When $u$ is a stationary harmonic map in $H^{2,2}(\mathbf{B}, N)$, this was shown, for example, in $[\mathbf{H}]$. Also one can show (5.1.1) by modifying the proof of the monotonicity identity in $[\mathbf{H L}]$ as follows. Starting with the last identity on page 570 in [HL] (for $p=2, r=1$ ), first take $R=1$ and $s \rightarrow 1$, then let $r \uparrow s=1$ (instead of $s \downarrow r)$. In this process, only that $\frac{\partial u}{\partial \mathbf{n}} \mid \mathbf{S}^{m-1} \in L^{2}\left(\mathbf{S}^{m-1}\right)$ is needed.

5.2. Lemma. Suppose that $u \in C^{1 ; 0}\left(\mathbf{B}_{0}, N\right)$ is a homogeneous harmonic map and $v \in H^{1}(\mathbf{B}, N)$ belongs to either of two classes in Lemma 5.1 which satisfies $v=u$ on $\mathbf{S}^{m-1}$ but $v \neq v$. Then

$$
E(v)<E(u)
$$

In particular, $u$ is the unique energy minimizer (or unique harmonic map) as long as u has least energy (among the harmonic maps).

Proof : By the assumption and Lemma 5.1 we have that (5.1.1) holds for $v$

$$
\int_{\mathbf{S}^{m-1}}\left|\nabla_{\tan } v\right|^{2}=\int_{\mathbf{S}^{m-1}}\left|\frac{\partial v}{\partial \mathbf{n}}\right|^{2}+(m-2) \int_{\mathbf{B}}|\nabla v|^{2} .
$$

Now note that since $v \neq u$, we must have that $\frac{\partial v}{\partial \mathbf{n}} \neq 0$, for otherwise we would have $v=u$ and $\frac{\partial v}{\partial \mathbf{n}}=\frac{\partial u}{\partial \mathbf{n}}$ on $\mathbf{S}^{m-1}$. By unique continuation theorem [ML2, Prop. 3.2], we have $v \equiv u$, a contradiction to that $v \neq u$. Thus we have $\int_{\mathbf{S}^{m-1}}\left|\frac{\partial v}{\partial \mathbf{n}}\right|^{2}>0$, which impies that

$$
\int_{\mathbf{B}}|\nabla v|^{2}<\frac{1}{m-2} \int_{\mathbf{S}^{m-1}}\left|\nabla_{\tan } v\right|^{2}=\frac{1}{m-2} \int_{\mathbf{S}^{m-1}}\left|\nabla_{\tan } u\right|^{2}=\int_{\mathbf{B}}|\nabla u|^{2} .
$$

This implies that if $u$ and $v$ both have least energy (or least energy among harmonic maps), then $v \equiv u$. 
5.3. Proposition. For any harmonic map $\omega: \mathbf{S}^{2} \rightarrow \mathbf{S}^{2}$, let

$$
u: \mathbf{B}^{3} \rightarrow \mathbf{S}^{2}, u(x)=\omega\left(\frac{x}{|x|}\right), x \in \mathbf{B}^{3} \backslash\{0\},
$$

be the corresponding homogeneous harmonic map. Then $u \in C^{2, \alpha ; 0}\left(\mathbf{B}_{0}^{3}, \mathbf{S}^{2}\right)$ for any $0<\alpha<1$. Furthermore, we have

(a). $\omega$ is stable.

$u$ is strictly stable with rate $\frac{1}{4}$.

(b). $\omega$ is energy minimizing among the maps having same degree as $\omega$ has.

$u$ is the unique energy minimizing among the maps in $C^{1 ; 0}\left(\mathbf{B}_{0}^{3}, \mathbf{S}^{2}\right)$ having same boundary data.

Proof : It is a well-known result in [BCL] that $\omega$ has least energy among the maps from $\mathbf{S}^{2}$ to itself having same degree. Consequently, $\omega$ is stable, since the continuity of degree implies that for any smooth variation $\omega_{t}$ of $\omega, \operatorname{deg}\left(\omega_{t}\right)=\operatorname{deg}(\omega)$ when $t$ is small, then $E\left(u_{t}\right) \geq E(u)$ and so $\left.\frac{d}{d t}\right|_{t=0} E\left(u_{t}\right) \geq 0$.

It follows from Lemma $4.2, u$ is strictly stable with rate $\frac{1}{4}$. To show that $u$ is strictly minimizing, let $v \in C^{1 ; 0}\left(\mathbf{B}_{0}^{3}, \mathbf{S}^{2}\right)$ with $v \mid \mathbf{S}^{2}=\omega$. Again by continuity of degree, $\operatorname{deg}(v(r \cdot))=\operatorname{deg}(\omega)$ for each $r>0$, thus by minimality of $\omega$,

$$
\begin{aligned}
\int_{\mathbf{B}^{3}}|\nabla v|^{2} & =\int_{0}^{1} r \int_{\mathbf{S}^{2}}\left|\nabla_{\mathbf{S}^{2}} v(r \cdot)\right|^{2} d \mathbf{S}^{2} d r+\int_{\mathbf{B}^{3}}\left|\frac{\partial v}{\partial r}\right|^{2} \\
& \geq \int_{0}^{1} r \int_{\mathbf{S}^{2}}\left|\nabla_{\mathbf{S}^{2}} \omega\right|^{2} d \mathbf{S}^{2} d r+\int_{\mathbf{B}^{3}}\left|\frac{\partial(v-u)}{\partial r}\right|^{2} \\
& \geq \frac{1}{2} \int_{\mathbf{S}^{2}}\left|\nabla_{\mathbf{S}^{2}} \omega\right|^{2} d \mathbf{S}^{2}+\frac{1}{4} \int_{\mathbf{B}^{3}}\left|\frac{v-u}{r}\right|^{2} \\
& =\int_{\mathbf{B}^{3}}|\nabla u|^{2}+\frac{1}{4} \int_{\mathbf{B}^{3}}\left|\frac{v-u}{r}\right|^{2} .
\end{aligned}
$$

5.4. Proposition. Let $\mathbf{I}: \mathbf{S}^{m-1} \rightarrow \mathbf{S}^{m-1}(m \geq 4)$ be the identity and $u: \mathbf{B}^{m} \rightarrow \mathbf{S}^{m-1}$ be the homogeneous extension, that is, $u(x)=\frac{x}{|x|}$. Then

(a). I is not stable for all $m \geq 4$. In fact, index $(\mathbf{I})=m$.

$u: \mathbf{B}^{m} \rightarrow \mathbf{S}^{m-1}$ is stable for all $m=3$ and strictly stable for $m=3$ and all $m \geq 5$.

(b). $u$ is the unique minimizer in $H^{1}\left(\mathbf{B}^{m}, \mathbf{S}^{m-1}\right)$ for all $m \geq 3$ and strictly minimizing for $m \geq 7$.

Proof : That index $(\mathbf{I})=m$ is computed by R. T. Smith [SRT]. That $u$ is the unique minimizer in $H^{1}\left(\mathbf{B}^{m}, \mathbf{S}^{m-1}\right)$ was proved in $[\mathbf{L F}]$ and $[\mathbf{J K}]$ and $[\mathbf{B C L}]$. The uniqueness can also be seen from Lemma 5.2 above. The stability and strict stability was shown in $[\mathbf{B A}]$.

Now we review the harmonic equator maps from the unit ball $\mathbf{B}^{m}$ to the spheroid $\mathbf{S}_{a}^{m}=\{(u, z) \in$ $\left.\mathbf{R}^{m+1}:|u|^{2}+\frac{|z|^{2}}{a^{2}}=1\right\}$, with $a>0$.

5.5. Proposition. Let $u_{*}: \mathbf{B}^{m} \rightarrow \mathbf{S}_{a}^{m},{ }_{*} u(x)=\left(\frac{x}{|x|}, 0\right)$, be the equator map. Then

(a). $u_{*}$ is stable for $a \geq \frac{2 \sqrt{m-1}}{m-2}$ and and $m \geq 3$;

$u$ is strictly stable for $m=3$ or $m \geq 5$ and $a>\frac{2 \sqrt{m-1}}{m-2}$.

If $a<\frac{2 \sqrt{m-1}}{m-2}$, then $u_{*}$ is unstable.

(b). If $a>\frac{2 \sqrt{m-1}}{m-2}$ and $m \geq 3$, then $u_{*}$ is strictly minimizing in rotationally symmetric maps.

If $m \geq 7$ and $a>\frac{2 \sqrt{m-1}}{m-2}$, then $u_{*}$ is strictly minimizing in $H^{1}\left(\mathbf{B}, \mathbf{S}_{a}^{m}\right)$.

Proof : These properties were proved in $[\mathbf{B A}]$ and $[\mathbf{M S}]$. The strictly stability also follows from Remark 3.3 and the Lemma 4.2 . 
Suppose $u \in C^{1 ; 0}\left(\mathbf{B}_{0}, N\right)$ is a homogeneous harmonic map with $\omega=u \mid \mathbf{S}^{m-1}$. Let $L=\operatorname{index}(\omega)+$ $\operatorname{nullity}(\omega)+1$, then $\mu_{L}$ is the first positive eigenvalue of $J_{\omega}$ and $\gamma_{L}=\frac{2-m}{2}+\sqrt{\frac{(m-2)^{2}}{4}+\mu_{L}}>0$. Let $\nu \in\left(0, \gamma_{L}\right)$ and $0<\alpha<1$. Recall that $C_{2}^{2, \alpha}\left(\mathbf{S}^{m-1}, T_{u} N\right)$ is the subspace of $C^{2, \alpha}\left(\mathbf{S}^{m-1}, T_{u} N\right)$ spaned by the eigenvectors of $\omega$ corresponding to positive eigenvalues. Combining the Theorem 4.7 and Theorem 3.8, we have the following

5.6 Theorem. There is a neighborhood $U$ of 0 in $C_{2}^{2, \alpha}\left(\mathbf{S}^{m-1}, T_{u} N\right)$ and a small number $\epsilon>0$ so that, for each $\varphi \in U$, there exists a harmonic map $v \in V_{\epsilon}=\left\{v \in C^{2, \alpha ; 0}\left(\mathbf{B}_{0}, N\right):\|v-u\|_{2, \alpha ; \nu}<\epsilon\right\}$ so that $v \mid \mathbf{S}^{m-1}=\pi(\omega+\varphi)$.

We can choose $U$ and $\epsilon$ to be small, so that

If $u$ is strictly stable, then $v$ is also strictly stable. Thus the harmonic maps in $V_{\epsilon}$ form a manifold.

If $u$ strictly minimizing, then $v$ is also strictly minimizing. Therefore, the harmonic maps in $V_{\epsilon}$ also form a manifold.

5.7. Remark. Note that strict minimality implies uniqueness, thus the strict minimality of $\omega$ implies the uniqueness of those $v$ with $v \mid \mathbf{S}^{m-1}$ close to $\omega$.

5.8. Remark. Proposition 5.6 applies especially to those maps in Propositions 5.3, 5.4 and 5.5.

\section{$\S$ 6. Global Structure of Smooth Harmonic Maps and Applications}

In this section we consider only smooth harmonic maps and obtain various results by modifying arguments of Brian White's treatment [WB] of minimal immersions. Theorem 6.3 shows that any Jacobi field in $C^{k, \alpha}\left(\Omega, T_{u} N\right)$ is integrable. Theorems 6.2 and 6.4 describe the local and global structures of the space $\mathcal{H}$ of the smooth harmonic maps.

6.1. Proposition. Suppose that $J_{u}: C^{k, \alpha}\left(\Omega, T_{u} N\right) \rightarrow C^{k-2, \alpha}\left(\Omega, T_{u} N\right)$ is the Jacobi operator with respect to a harmonic map $u \in C^{k, \alpha}(\Omega, N)$. Let $K_{0}$ be the kernel of $J_{u}$ in $C_{0}^{k, \alpha}\left(\Omega, T_{u} N\right)$ and $\left[K_{0}\right]^{\perp}$ is orthogonal complement in $L^{2}\left(\Omega, T_{u} N\right)$. Then

$$
\operatorname{dim}\left(K_{0}\right)<\infty, \quad \operatorname{Im} J_{u}=\left[K_{0}\right]^{\perp} \cap C^{k-2, \alpha}\left(\Omega, T_{u} N\right) .
$$

So $J_{u}$ is Fredholm of index 0.

Proof. Being considered as an operator defined on $H_{0}^{1}\left(\Omega, T_{u} N\right), J_{u}$ is self-adjoint (by (1.7.1)) and uniformly elliptic. Furthermore by standard elliptic theory any solution $\kappa$ of $J_{u} \kappa=f$ for $f \in C^{k-2, \alpha}\left(\Omega, T_{u} N\right)$ is in $C^{k, \alpha}\left(\Omega, T_{u} N\right)$ and

$$
\|\kappa\|_{k, \alpha} \leq C\left[\|I \kappa\|_{k-2, \alpha}+\left\|J_{u} \kappa\right\|_{k-2, \alpha}\right],
$$

where $I: C_{0}^{k, \alpha}\left(\Omega, T_{u} N\right) \rightarrow C^{k-2, \alpha}\left(\Omega, T_{u} N\right)$ is the inclusion, which is compact. This inequality implies that $J_{u}$ has closed range, by [T Proposition 3.1]. Thus $K_{0}$ is equal to the kernel of $J_{u}$ in $H_{0}^{1}\left(\Omega, T_{u} N\right)$ which is finitely dimensional. By Fredholm alternative, $J_{u} \kappa=f$ is solvable iff $f \in K_{0}^{\perp}$. So $\operatorname{Im} J_{u}=\left[K_{0}\right]^{\perp} \cap C^{k-2, \alpha}\left(\Omega, T_{u} N\right)$.

6.2. Theorem (Local Structure). Suppose $u \in \mathcal{H}$ and $K_{0}=K_{0}\left(J_{u}\right)$. Then there exist smooth maps

$$
\begin{aligned}
F & : C^{k, \alpha}(\partial \Omega, N) \times K_{0} \rightarrow C^{k, \alpha}(\Omega, N), \\
g & : C^{k, \alpha}(\partial \Omega, N) \times K_{0} \rightarrow K_{0},
\end{aligned}
$$


defined on some neighborhood $U=U_{1} \times U_{2}$ of $(\varphi, 0), \varphi=u \mid \partial \Omega$, and satisfying the following:

(a). $F(u \mid \partial \Omega, 0)=u$.

$F(\psi, \kappa) \mid \partial \Omega=\psi$ for all $(\psi, \kappa) \in U$.

$F(\psi, \kappa)$ is harmonic if and only if $g(\psi, \kappa)=0$.

(b). $D_{2} F(\varphi, 0) \mid K_{0}=i d$.

$D F(\varphi, 0): C^{k, \alpha}\left(\partial \Omega, T_{\varphi} N\right) \times K_{0} \rightarrow C^{k, \alpha}\left(\Omega, T_{u} N\right)$ is an immersion.

(c). $D g(\varphi, 0): C^{k, \alpha}\left(\partial \Omega, T_{\varphi} N\right) \times K_{0} \rightarrow K_{0}$ is a submersion.

(d). For every $\varepsilon>0$, there is a neighborhood $W$ of $u$ in $C^{k, \alpha}(\Omega, N)$ such that each harmonic map $v$ in $W$ equals $F(v \mid \partial \Omega, \kappa)$ for some $\kappa \in K_{0}$ with $\|\kappa\|_{k, \alpha}<\varepsilon$.

(e). $U$ and $W$ can be chosen so that $\mathcal{U}=U \cap g^{-1}(0)$ and $\mathcal{W}=W \cap \mathcal{H}$ are smooth submanifolds of $C^{k, \alpha}\left(\partial \Omega, T_{\varphi} N\right) \times K_{0}$ and $C^{k, \alpha}(\Omega, N)$ respectively.

$F: \mathcal{U} \rightarrow \mathcal{W}$ is an isomorphism and $\Pi \circ F(\psi, \kappa)=\psi$.

$\mathcal{U}$ has codimension $\operatorname{dim} K_{0}$ and its tangent space at $(\varphi, 0)$ is

$$
T_{(\varphi, 0)} \mathcal{U}=\operatorname{Ker} D_{1} g(\varphi, 0) \oplus K_{0}
$$

(f). For $0<\alpha^{\prime}<\alpha, U$ and $W$ can be replaced by $U^{\prime}$ and $W^{\prime}$ that are open, with respect to the corresponding $C^{k, \alpha^{\prime}}$ norms, in $C^{k, \alpha}(\partial \Omega, N)$ and $C^{k, \alpha}(\Omega, N)$, respectively.

Remark. It is perhaps useful to check the analogue of this theorem in the well-known elementary case of geodesic arcs $u:[0,1] \rightarrow N$. Here the association of the initial position and initial velocity of a geodesic gives a global diffeomorphism between $\mathcal{H}$ and the tangent bundle of $N$. As above, each geodesic $u \in \mathcal{H}$ has a neighborhood diffeomorphic to a 2n-dimensional submanifold of a neighborhood of $(0, u(0), u(1))$ in $K_{0} \times N \times N$.

\section{Proof of Theorem 6.2.}

Proof of (a): Construction of $F$ and $g$. Denote $K_{0}^{\perp}=\left[K_{0}^{\perp}\right] \cap C^{k-2, \alpha}\left(\Omega, T_{u} N\right)$ and $K_{0}^{\prime}=K_{0}^{\perp} \cap$ $C_{0}^{k, \alpha}\left(\Omega, T_{u} N\right)$ and $P_{K_{0}^{\perp}}: L^{2}\left(\Omega, T_{u} N\right) \rightarrow K_{0}^{\perp}$ the orthogonal projection. For $\psi \in C^{k, \alpha}(\partial \Omega, N)$, let $\bar{\psi}$ be the unique function satisfying

$$
\begin{cases}\Delta \bar{\psi}=0, & \text { on } \Omega \\ \bar{\psi}=\psi-\varphi, & \text { on } \partial \Omega\end{cases}
$$

Let $\Phi(\varphi)=P_{u} \circ \bar{\psi}$, the projection to $T_{u} N$. Clearly, $\Phi(\varphi)=0$, and $\Phi(\psi) \in C^{k, \alpha}\left(\Omega, \mathbf{R}^{p}\right)$. By Schauder's estimates, if $\psi$ is $C^{k, \alpha}(\partial \Omega, N)$ close to $\varphi$, then $\Phi(\psi) \in C^{k, \alpha}\left(\Omega, \mathbf{R}^{p}\right)$ is close to 0 .

Define

$$
\begin{array}{r}
\Theta: C^{k, \alpha}(\partial \Omega, N) \times K_{0} \times K_{0}^{\prime} \rightarrow K_{0}^{\perp}, \\
\Theta(\psi, \kappa, \eta)=P_{K_{0}^{\perp}} \circ P_{u} \circ H[\pi(u+\Phi(\psi)+\kappa+\eta)] .
\end{array}
$$

Then from Proposition 6.1, we have $D_{3} \Theta(\varphi, 0,0)=P_{K_{0}^{\perp}} \circ P_{u} \circ D H\left|K_{0}^{\prime}=J_{u}\right| K_{0}^{\prime}$, which is isomorphism. By the impicit function theorem, there are neighborhoods $U=U_{1} \times U_{2}$ of $(\varphi, 0)$ in $C^{k, \alpha}(\partial \Omega, N) \times K_{0}, V$ of 0 in $K_{0}^{\prime}$, and a smooth map

$$
Q: U \subseteq C^{k, \alpha}(\partial \Omega, N) \times K_{0} \rightarrow K_{0}^{\prime}
$$

such that for $(\psi, \kappa) \in U, \eta=Q(\psi, \kappa)$ is the unique solution in $V$ of $\Theta(\psi, \kappa, \eta)=0$. Let

$$
\begin{aligned}
& F(\psi, \kappa)=\pi(u+\Phi(\psi)+\kappa+Q(\psi, \kappa)), \\
& g(\psi, \kappa)=P_{K_{0}} \circ P_{u} \circ H(F(\psi, \kappa)) .
\end{aligned}
$$


Then

$$
\begin{aligned}
& \left.\Theta(\psi, \kappa, Q(\psi, \kappa))=P_{K_{0}^{\perp}} \circ P_{u} \circ H[F(\psi, \kappa))\right]=0 . \\
& g(\psi, \kappa)=P_{u} \circ H(F(\psi, \kappa)), .
\end{aligned}
$$

Now that $F$ and $g$ satisfy (a) follows from their definitions and (4.7.3).

Proof of (b). Let $\kappa \in K_{0}$. By definition of $F$,

$$
D_{2} F(\varphi, 0) \kappa=\kappa+D_{2} Q(\varphi, 0) \kappa .
$$

Differentiating

$$
P_{K_{0}^{\perp}} \circ P_{u} \circ H[\pi(u+t \kappa+Q(\varphi, t \kappa))]=0
$$

for $t$ at $t=0$, we get

$$
\begin{aligned}
0 & =\left\langle\kappa+D_{2} Q(\varphi, 0) \kappa, J_{u}\right\rangle \\
& =\left\langle D_{2} Q(\varphi, 0) \kappa, J_{u}\right\rangle .
\end{aligned}
$$

So $D_{2} Q(\varphi, 0) \kappa \in K_{0}$. On the other hand, the image of $Q$ is in $K_{0}^{\perp}$, therefore

$$
D_{2} Q(\varphi, 0) \kappa \in K_{0} \cap K_{0}^{\perp}=\{0\} .
$$

Thus $D_{2} F(\varphi, 0) \kappa=\kappa$.

To show that $D F(\varphi, 0)$ is an immersion, let $(\xi, \kappa) \in C^{k, \alpha}\left(\partial \Omega, T_{\varphi} N\right) \times K_{0}$, then

$$
D F(\varphi, 0)(\xi, \kappa)=\xi_{*}+\kappa, \quad \text { where } \xi_{*}=D_{1} F(\varphi, 0) \xi \text {. }
$$

Now look at the following sequence

$$
\begin{aligned}
C^{k, \alpha}\left(\partial \Omega, T_{\varphi} N\right) \times K_{0} & \stackrel{D F(\varphi, 0)}{\longrightarrow} C^{k, \alpha}\left(\Omega, T_{u} N\right) \\
& \stackrel{\Lambda}{\longrightarrow} C^{k, \alpha}\left(\partial \Omega, T_{\varphi} N\right) \times C_{0}^{k, \alpha}\left(\Omega, T_{u} N\right) \stackrel{P r_{1}}{\longrightarrow} C^{k, \alpha}\left(\partial \Omega, T_{\varphi} N\right) \times K_{0},
\end{aligned}
$$

where $\Lambda \xi=\left(\xi \mid \partial \Omega, \xi-\xi_{*}\right)$ is an isomorphism, as one can check easily. The composition $\operatorname{Pr}_{1} \circ \Lambda \circ D F(\varphi, 0)=$ $i d$, so $\operatorname{DF}(\varphi, 0)$ is an immersion $[\mathbf{L}, \mathrm{II}, 2]$.

Proof of (c). It suffices to show that $D g(\varphi, 0): C^{k, \alpha}\left(\partial \Omega, T_{\varphi} N\right) \times K_{0} \rightarrow K_{0}$ is surjective. First note that for $(\xi, \kappa) \in C^{k, \alpha}\left(\partial \Omega, T_{\varphi} N\right) \times K_{0}$, by definition of $g$ and $(6.2 .7)$,

$$
D g(\varphi, 0)(\xi, \kappa)=D_{1} g(\varphi, 0) \xi=J_{u} \xi_{*} .
$$

Secondly note that

$$
V_{u \mid \partial \Omega}=\left\{\frac{\partial \kappa}{\partial \mathbf{n}}: \kappa \in K_{0}\right\}
$$

is a subspace of $C^{k, \alpha}\left(\partial \Omega, T_{\varphi} N\right)$ of dimension $\operatorname{dim}\left(K_{0}\right)$. This is because if $0 \neq \kappa \in K_{0}$, then $k \mid \partial \Omega=0$ implies $\frac{\partial \kappa}{\partial \mathbf{n}} \in C^{k, \alpha}\left(\partial \Omega, T_{\varphi} N\right)$, and Calderon's uniqueness theorem implies that $0 \neq \frac{\partial \kappa}{\partial \mathbf{n}}([\mathbf{C 1}],[\mathbf{M L 2}$, Prop.3.2].).

Thus to show $\operatorname{Dg}(\varphi, 0)$ is surjective, it is enough to show that

$$
D_{1} g(\varphi, 0): V_{u \mid \partial \Omega} \rightarrow K_{0}
$$

is one-to-one. Indeed, by (6.2.8),

$$
D_{1} g(\varphi, 0) \frac{\partial \kappa}{\partial \mathbf{n}}=J_{u} \eta, \quad \eta=\left(\frac{\partial \kappa}{\partial \mathbf{n}}\right)_{*}
$$

Thus, if $D_{1} g(\varphi, 0) \frac{\partial \kappa}{\partial \mathbf{n}}=0$, then $\eta$ is a Jacobi field with $\eta=\frac{\partial \kappa}{\partial \mathbf{n}}$ on $\partial \Omega$. By (1.6.1) and symmetry in $\eta$ and $\kappa$, we will have

$$
0=\int_{\Omega}-\kappa \cdot J_{u} \eta=\int_{\Omega}-J_{u} \kappa \cdot \eta+\int_{\partial \Omega} \frac{\partial \kappa}{\partial \mathbf{n}} \cdot \eta=\int_{\partial \Omega}\left|\frac{\partial \kappa}{\partial \mathbf{n}}\right|^{2},
$$

and so $\frac{\partial \kappa}{\partial \mathbf{n}} \equiv 0$. So $D_{1} g(\varphi, 0)$ is one-to-one. 
Proof of $(d)$. Suppose that $v$ is a smooth harmonic map close to $u$.

Let $\lambda(x) \in T_{u(x)} N$ be the unique vector such that $\pi(u(x)+\lambda(x))=v(x)$. Then $\lambda \in C^{k, \alpha}\left(\Omega, T_{u} N\right)$. Let $h=\Phi(\psi)-\lambda$. Then

$$
v=\pi(u+\Phi(\psi)+h)
$$

Let $\kappa=P_{K_{0}} h, \eta=h-\kappa \in K_{0}^{\perp}$. Then

$$
v=\pi(u+\Phi(\psi)+\kappa+\eta) .
$$

From the fact that $v$ is harmonic, we have

$$
0=P_{K_{0}^{\perp}} \circ P_{u} \circ H[\pi(u+\Phi(\psi)+\kappa+\eta)]=\Theta(\psi, \kappa, \eta),
$$

which has a unique solution $\eta=Q(\psi, \kappa)$. Thus by (6.2.11) and the definition of $F, v=(F(\psi, \kappa))$.

Proof of $(e)$. That $D g(\varphi, 0)$ is an immersion implies that $g^{-1}(0)$ is a smooth submanifold near $(\varphi, 0)$ of codimension $\operatorname{dim}\left(K_{0}\right)[\mathbf{L}$, II,2]. By (b), (d) and again $[\mathbf{L}]$, we may choose $U$ and $W$ suitably small so that $\mathcal{U}=U \cap g^{-1}(0), F(\mathcal{U})$ and $\mathcal{W}$ are all smooth manifolds, and $F: \mathcal{U} \rightarrow \mathcal{W}$ is an isomorphism. By (6.2.8), the tangent space to $\mathcal{U}$ at $(\varphi, 0)$ is

$$
T_{(\varphi, 0)} \mathcal{U}=\operatorname{Ker} D_{1} g(\varphi, 0) \oplus K_{0} .
$$

Proof of (f). Noting that $u \in C^{k, \alpha}(\Omega, N) \subseteq C^{k, \alpha^{\prime}}(\Omega, N)$, we may apply (a) and (b) of the Theorem to $u$ with $C^{k, \alpha}(\Omega, N)$ replaced by $C^{k, \alpha^{\prime}}(\Omega, N)$. In this way, we get the corresponding neighborhoods $U^{\prime \prime}$ of $u \mid \partial \Omega$ in $C^{k, \alpha^{\prime}}(\partial \Omega, N), W^{\prime \prime}$ of $u$ in $C^{k, \alpha^{\prime} ; 0}\left(\Omega_{0}, N\right)$ and a smooth map

$$
F^{\prime}: U^{\prime \prime} \subseteq C^{k, \alpha^{\prime}}(\partial \Omega, N) \rightarrow C^{k, \alpha^{\prime}}(\Omega, N)
$$

We first show

$$
F^{\prime}: U^{\prime \prime} \cap C^{k, \alpha}(\partial \Omega, N) \rightarrow C^{k, \alpha}(\Omega, N)
$$

is smooth and $F^{\prime}\left|U^{\prime \prime} \cap U=F\right| U^{\prime \prime} \cap U$.

Indeed, if $\psi \in U^{\prime \prime} \cap C^{k, \alpha}(\partial \Omega, N)$, then $F^{\prime}(\psi) \in C^{k, \alpha^{\prime}}(\Omega, N)$ is harmonic, that is, it satisfies

$$
\begin{cases}H\left(F^{\prime}(\psi)\right)=\Delta F^{\prime}(\psi)-A\left(F^{\prime}(\psi)\right)\left(d F^{\prime}(\psi), d F^{\prime}(\psi)\right)=0 & \text { on } \Omega, \\ F^{\prime}(\psi)=\psi & \text { on } \partial \Omega .\end{cases}
$$

Since $A\left(F^{\prime}(\psi)\right)\left(d F^{\prime}(\psi), d F^{\prime}(\psi)\right) \in C^{k-1, \alpha^{\prime}}\left(\Omega, \mathbf{R}^{p}\right) \subseteq C^{k-2, \alpha}\left(\Omega, \mathbf{R}^{p}\right)$ (see $\left.1.2(\mathrm{e})\right)$ and $\psi \in C^{k, \alpha}(\partial \Omega, N)$, it follows from Theorem 1.10 that $F^{\prime}(\psi) \in C^{k, \alpha}(\Omega, N)$.

Furthermore, it follows from definitions that $Q^{\prime}(\psi)=Q(\psi)$ on $U^{\prime \prime} \cap U$, and then, $F=F^{\prime}$ on $U^{\prime \prime} \cap U$. This also implies the smoothness of $F^{\prime}$ with respect to $C^{k, \alpha}$ norms.

Now we see that one may replace the $F$ in (a) and (b) by $F^{\prime}$, whose domain $U^{\prime \prime} \cap C^{k, \alpha}(\partial \Omega, N)$ is open in $C^{k, \alpha}(\partial \Omega, N)$ with respect to norm $\|\cdot\|_{k, \alpha^{\prime}}$. To end the proof of (c), set $U^{\prime}=U^{\prime \prime} \cap C^{k, \alpha}(\partial \Omega, N)$ and $W^{\prime}=W^{\prime \prime} \cap C^{k, \alpha}(\Omega, N)$.

Denote $K=K\left(J_{u}\right)=\left\{\kappa \in C^{k, \alpha}\left(\Omega, T_{u} N\right): J_{u} \kappa=0\right\}$, and $K_{0}=K_{0}\left(J_{u}\right)=\left\{\kappa \in C_{0}^{k, \alpha}\left(\Omega, T_{u} N\right): J_{u} \kappa=\right.$ $0\}$. Again, a $\kappa \in K$ is called a Jacobi field. We have

6.3. Theorem. $\xi \in C^{k, \alpha}\left(\Omega, T_{u} N\right)$ is a Jacobi field to $u \in \mathcal{H}$ if and only if $\xi$ is the initial velocity vector field of a one-parameter family of harmonic maps. 
Proof : Suppose $\xi \in K\left(J_{u}\right)$. Let

$$
\xi_{*}=: D_{1} F(u \mid \partial \Omega, 0)(\xi \mid \partial \Omega) .
$$

Then by Theorem $6.2(\mathrm{a}), \xi_{*}|\partial \Omega=\xi| \partial \Omega$, and we now show $\xi_{*} \in K\left(J_{u}\right)$. Indeed, by differentiating the identity $\left.0=P_{K_{0}^{\perp}} \circ P_{u} \circ H[F(\pi(u|\partial \Omega+t \xi| \partial \Omega), 0))\right]$, we get

$$
0=P_{K_{0}^{\perp}}\left\langle\xi_{*}, P_{u} \circ D H(u)\right\rangle=P_{K_{0}^{\perp}}\left(J_{u} \xi_{*}\right) .
$$

On the other hand $J_{u} \xi_{*}=J_{u}\left(\xi_{*}-\xi\right) \in \operatorname{Im} J_{u}=K_{0}^{\perp}$. So $J_{u} \xi_{*}=0$.

Now let $\kappa=\xi-\xi_{*}$, then $\kappa \in K_{0}=K_{0}\left(J_{u}\right)$. From (6.2.8), (6.2.12) we know that $(\xi \mid \partial \Omega, \kappa)$ is in the tangent space to $g^{-1}(0)$ at $(u \mid \partial \Omega, 0)$. It follows from Theorem 6.2 (e) that there is a one-parameter family $\left(\psi_{t}, \kappa_{t}\right)$ in $C^{k, \alpha}(\partial \Omega, N) \times K_{0}$ satisfying

$$
\begin{aligned}
\left(\psi_{0}, \kappa_{0}\right) & =(u \mid \partial \Omega, 0), \\
\left.\frac{d}{d t}\right|_{t=0}\left(\psi_{t}, \kappa_{t}\right) & =(\xi \mid \partial \Omega, \kappa)
\end{aligned}
$$

such that $g\left(\psi_{t}, \kappa_{t}\right)=0$. Thus Theorem 6.2 (a) implies that $F\left(\psi_{t}, \kappa_{t}\right)$ is a one-parameter family of harmonic maps, which has initial velocity vector field:

$$
\left.\frac{d}{d t}\right|_{t=0} F\left(\psi_{t}, \kappa_{t}\right)=\xi_{*}+\kappa=\xi .
$$

Conversely, suppose $\xi=\left.\frac{d}{d t}\right|_{t=0} u_{t}$ with $u_{t} \in \mathcal{H}$ and $u_{0}=u$. Then $H\left(u_{t}\right)=0$ implies $J_{u} \xi=\left.\frac{d}{d t}\right|_{t=0} H\left(u_{t}\right)=$ 0 . That is, $\xi \in K\left(J_{u}\right)$.

\subsection{Theorem (Global Structure).}

(a). $\mathcal{H}$ is a smooth Banach manifold modelled on $C^{k, \alpha}(\partial \Omega, N)$, with a countable cover of coordinate charts given in Theorem $6.2(e)$ and $(f)$.

(b). The tangent space to $\mathcal{H}$ at $u \in \mathcal{H}$ is $T_{u} \mathcal{H}=K\left(J_{u}\right) . V_{u \mid \partial \Omega}=\left\{\frac{\partial \kappa}{\partial \mathbf{n}}: \kappa \in K_{0}\left(J_{u}\right)\right\}$ is a subspace of $C^{k, \alpha}\left(\partial \Omega, T_{u \mid \partial \Omega} N\right)$ with $\operatorname{dim}\left(V_{u \mid \partial \Omega}\right)=\operatorname{dim}\left(K_{0}\left(J_{u}\right)\right)$ and perpendicular to $D \Pi\left(T_{u} \mathcal{H}\right)$, where $\Pi: \mathcal{H} \rightarrow$ $C^{k, \alpha}(\partial \Omega, N)$ in the projection.

(c). The map $\Pi: \mathcal{H} \rightarrow C^{k, \alpha}(\partial \Omega, N)$ is Fredholm of index 0 with $\operatorname{Ker} D \Pi(u)=K_{0}\left(J_{u}\right)$. So $D \Pi(u)$ is onto if and only if $K_{0}\left(J_{u}\right)=\{0\}$.

Proof : The set $\mathcal{A}$ of all such pairs $(\mathcal{W}, \Gamma)\left(\Gamma=F^{-1}\right)$ in Theorem 6.2 (e) (f) form an atlas for $\mathcal{H}$. To verify the smoothness of the transition maps, let $\left(\mathcal{W}_{1}, \Gamma_{1}\right),\left(\mathcal{W}_{2}, \Gamma_{2}\right)$ be two charts corresponding to $F_{1}$ and $F_{2}$, then

$$
\left.\Gamma_{1} \circ \Gamma_{2}^{-1}\right|_{\Gamma_{2}\left(\mathcal{W}_{1} \cap \mathcal{W}_{2}\right)}=\left.\Gamma_{1} \circ F_{2} \circ\left(\Gamma_{2} \circ F_{2}\right)^{-1}\right|_{\Gamma_{2}\left(\mathcal{W}_{1} \cap \mathcal{W}_{2}\right)},
$$

is smooth. By the separability of $\mathcal{H}$ with respect to $\|\cdot\|_{k, \alpha^{\prime}, 0}$ with $0<\alpha^{\prime}<\alpha, \mathcal{A}$ contains a countable subcover. This shows (a).

The first part of (b) is just Theorem 6.3. To show the second part, let $\kappa \in K_{0}\left(J_{u}\right)$ and $\xi \in T_{u} \mathcal{H}$. Then by symmetry of the second variational formula (1.6.1) for $\xi, \kappa$, and the facts $J_{u} \xi=J_{u} \kappa=0$, we get

$$
\int_{\partial \Omega} \frac{\partial \kappa}{\partial \mathbf{n}} \cdot \xi=\int_{\partial \Omega} \frac{\partial \xi}{\partial \mathbf{n}} \cdot \kappa=0 .
$$

That is, $V_{u \mid \partial \Omega} \perp T_{u} \mathcal{H}$. As shown in (6.2.9), $\operatorname{dim}\left(V_{u \mid \partial \Omega}\right)=\operatorname{dim}\left(K_{0}\left(J_{u}\right)\right)$.

Now we show (c). Note that for $\kappa \in T_{u} \mathcal{H}$,

$$
\langle\kappa, D(\Pi \mid \mathcal{H})(u)\rangle=\kappa \mid \partial \Omega .
$$

Thus Ker $D(\Pi \mid \mathcal{H})(u)=K_{0}\left(J_{u}\right)$, and $\operatorname{Im} D(\Pi \mid \mathcal{H})(u)=K\left(J_{u}\right) / K_{0}\left(J_{u}\right)$. By definition, $\Pi \mid \mathcal{H}$ is Fredholm of index 0 .

If $D(\Pi \mid \mathcal{H})(u)$ is onto, then by (b), $V_{u \mid \partial \Omega}=\{0\}$, so $K_{0}\left(J_{u}\right)=\{0\}$. The converse is obvious. 
6.5. Definition. A boundary map $\omega \in C^{k, \alpha}(\partial \Omega, N)$ is a regular value of $\Pi$ if $D(\Pi \mid \mathcal{H})(u)$ is surjective for all $u \in \mathcal{H} \cap \Pi^{-1}\{\omega\}$. This means that each such $u$ has no nonzero Jacobi fields that vanish on $\partial \Omega$ (Theorem $6.4(\mathrm{c}))$. A nonregular value is also called singular.

6.6. Theorem. The set of singular values of $\Pi$ is of first (Baire) category in $C^{k, \alpha}(\partial \Omega, N)$.

Proof : For an open subset $\mathcal{W} \subseteq \mathcal{H}$, we define $\mathcal{S}(\mathcal{W})$, where

$$
\mathcal{S}(\mathcal{W})=\left\{\omega \in C^{k, \alpha}(\partial \Omega, N): \omega \text { is a singular value of } \Pi \mid \mathcal{W}\right\}
$$

Since $\mathcal{H}$ is separable with respect to $C^{k, \alpha^{\prime}}$ norm $\left(0<\alpha^{\prime}<\alpha\right)$, to prove the theorem, it suffices to show that, for each $u \in \mathcal{H}$, there is a $C^{k, \alpha^{\prime}}$ open neighborhood $\mathcal{W}$ of $u$ in $\mathcal{H}$, so that $\mathcal{S}(\mathcal{W})$ is closed and nowhere dense.

For $u \in \mathcal{H}$, let $\mathcal{W}$ be chosen as in Theorem 6.2 (e) such that $\mathcal{W}$ can be identified as a codimension $\operatorname{dim}\left(K_{0}\right)$ submanifold of $U \times K_{0} .\left(K_{0}=K_{0}\left(J_{u}\right)\right.$.)

Clearly $\mathcal{S}(\mathcal{W})$ is closed. Now we prove that it is also nowhere dense, that is, any $\omega \in \mathcal{S}(\mathcal{W})$ can be approximated by an $\varphi \notin \mathcal{S}(\mathcal{W})$. Let

$$
\mathcal{V}=\mathcal{W} \cap\left(\exp _{\omega} V \times K_{0}\right)
$$

where $V=V_{u \mid \partial \Omega}=\left\{\frac{\partial \kappa}{\partial \mathbf{n}}: \kappa \in K_{0}\left(J_{u}\right)\right\}$ and and $\exp _{\omega}$ is the exponential map (as in $1.2(\mathrm{~b})$ ). Then $\mathcal{V}$ is a submanifold of $\exp _{\omega} V \times K_{0}$ of dimension and codimension $\operatorname{dim}\left(K_{0}\right)$. (cf. Theorem 6.4 (b).) Note that $\varphi \in \exp _{\omega} V$ is a regular value of $\Pi \mid \mathcal{V}$ if and only if it is a regular value of $\Pi$.

Consider the $\Pi: \mathcal{V} \subseteq \exp _{\omega} V \times K_{0} \rightarrow \exp _{\omega} V$. By Sard's Theorem, there is a regular value $z \in V$ of $\exp _{\omega}^{-1} \Pi$ that is arbitrarily close to 0 . Thus $\varphi=\exp _{\omega} z$ is a regular value of $\Pi \mid \mathcal{V}$, which can be arbitrarily close to $\omega$.

As an application of Theorem 6.2 and Theorem 6.4, here we prove some finiteness and uniqueness results for harmonic maps analogous to $[\mathbf{W B}]$. By Theorem 6.6, the following result can be considered as generic local finiteness.

6.7. Theorem. Suppose that $\omega$ is a regular value of $\Pi$. For any $M>0$, there exists a $C^{k, \alpha}$ neighborhood $U$ of $\omega$ so that each $\varphi \in U$ serves as boundary data for only finitely many harmonic maps $u \in C^{k, \alpha}(\Omega, N)$ with $\|u\|_{k, \alpha} \leq M$. The number of these harmonic maps is bounded independent of $\varphi \in U$.

Proof : Let $\alpha^{\prime}$ and $X$ be in the proof of Corollary 6.7. By Theorem 6.1 (a) each harmonic map $u \in$ $C^{k, \alpha}(\Omega, N)$ has a $C^{k, \alpha}$ neighborhood $W_{u}^{\prime}$ so that $\Pi$ maps the set of harmonic maps in $W_{u}^{\prime}$ diffeomorphically onto a $C^{k, \alpha^{\prime}}$ neighborhood $U^{\prime}$ of $\omega$ in $C^{k, \alpha}(\partial \Omega, N)$. Since $X$ is $C^{k, \alpha^{\prime} ; 0}$ compact, $X_{\omega}=: X \cap \Pi^{-1}\{\omega\}$ is finite, $V=\cap_{u \in X_{\omega}} U_{u}^{\prime}$ is $C^{k, \alpha^{\prime}}$ open, and

$$
\delta=\operatorname{dist}_{C^{k, \alpha^{\prime}}}\left(\Pi^{-1}\{\omega\},\left(X \backslash \Pi^{-1}(V)\right) \cap \cup_{u \in X_{\omega}} W_{u}^{\prime}\right)
$$

is positive. So we may choose a $C^{k, \alpha^{\prime}}$ open neighborhood $U$ about $\omega$ in

$$
V \backslash \Pi\left(X \cap\left\{u: \operatorname{dist}_{C^{k, \alpha^{\prime}}}\left(u, \Pi^{-1}\{\omega\}\right) \geq \delta\right\}\right) .
$$

Let $\varphi \in U$. As in the proof of 6.7, each harmonic $v \in C^{k, \alpha}(\Omega, N)$ with $v \mid \partial \Omega=\varphi$ and $\|v\|_{k, \alpha} \leq M$ must belong to $X_{\varphi}=X \cap \Pi^{-1}\{\varphi\}$. Thus the number of such $v$ 's is bounded by $\operatorname{card}\left(X_{\varphi}\right) \leq \operatorname{card}\left(X_{\omega}\right)$. 
As in [WB] we say that a subset $A$ of a Banach manifold $X$ is of codimension $c$ if $A \subseteq \cup_{i=1}^{\infty} \Pi_{i}\left(M_{i}\right)$ where $M_{i}$ is a codimension $c+k_{i}$ submanifold of $X \times \mathbf{R}^{k_{i}}$ and $\Pi_{i}$ is the projection map of $X \times \mathbf{R}^{k_{i}}$ onto $X$. On the space $C^{k, \alpha}(\partial \Omega, N)$, there are two other notions available: measure 0 (cf. [MF2][ML2]) and first category, both are weaker than codimension 1 ([WB, 1.7]). In [ML2], a uniqueness result for energy minimizers is proved in the sense of measure 0 .

6.8. Theorem.. The set of all regular values of $\Pi$ in $C^{k, \alpha}(\partial \Omega, N)$ that serves as boundary value of two or more harmonic maps in $C^{k, \alpha}(\Omega, N)$ having the same energy is a codimension 1 subset of $C^{k, \alpha}(\partial \Omega, N)$.

Proof : First note that the set of regular values of $\Pi$ is an open and dense subset of $C^{k, \alpha}(\partial \Omega, N)$. Denote

$$
\begin{aligned}
& \mathcal{C}=\left\{\left(u_{1}, u_{2}\right): u_{1}, u_{2} \in C^{k, \alpha}(\Omega, N)\right. \text { are harmonic maps with } \\
& \left.u_{1}\left|\partial \Omega=u_{2}\right| \partial \Omega \text { being regular, } u_{1} \neq u_{2} \text { and } E\left(u_{1}\right)=E\left(u_{2}\right)\right\} .
\end{aligned}
$$

Let $\left(u_{1}, u_{2}\right) \in \mathcal{C}$ with $u_{i} \mid \partial \Omega=\varphi$. For $i=1,2$, let $F_{i}$ be the maps in Theorem 6.1 corresponding to $u_{i}$, defined on neighborhood $U_{i}$ of $\varphi$ in $C^{k, \alpha}(\partial \Omega, N)$, which can be chosen to be open in the $C^{k, \alpha^{\prime}}$ norm.

Let $U=U_{1} \cap U_{2}$ and consider the function

$$
G: U \subseteq C^{k, \alpha}(\partial \Omega, N) \rightarrow \mathbf{R}
$$

defined by

$$
G(\psi)=\int_{\Omega}\left|\nabla F_{1}(\psi)\right|^{2}-\int_{\Omega}\left|\nabla F_{2}(\psi)\right|^{2} .
$$

For any $\psi \in U$, the map $v_{i}=F_{i}(\psi)$ is harmonic $(i=1,2)$, and for $h \in T_{u} U=C^{k, \alpha}\left(\partial \Omega, T_{\varphi} N\right)$, by first variational formula 1.4.1,

$$
\langle h, D G(\varphi)\rangle=\left.\frac{d}{d t}\right|_{t=0} G(\pi(\psi+t h))=\int_{\partial \Omega} h \cdot\left(\frac{\partial u_{1}}{\partial \mathbf{n}}-\frac{\partial u_{2}}{\partial \mathbf{n}}\right) .
$$

Since $u_{1} \neq u_{2}$, by a uniqueness continuation theorem in [ML2, Proposition 6.2], we have $\frac{\partial u_{1}}{\partial \mathbf{n}} \neq \frac{\partial u_{2}}{\partial \mathbf{n}}$. This implies that $D G(\varphi) \neq 0$.

By implicit function theorem, $G^{-1}(0) \cap U$ is a submanifold of $U$ of codimension 1.

By Theorem 6.1 (a) there is a small $\delta\left(u_{1}, u_{2}\right)>0$ such that if $\left(v_{1}, v_{2}\right) \in B\left(u_{1}, u_{2}\right)=:\left\{\left(v_{1}, v_{2}\right) \in \mathcal{C}\right.$ : $\left.\left\|v_{i}-u_{i}\right\|_{k, \alpha^{\prime} ; 0}<\delta\left(u_{1}, u_{2}\right), i=1,2\right\}$, then $v_{i}=F_{i}(\psi)$ and $\psi=v_{1}\left|\partial \Omega=v_{2}\right| \partial \Omega \in U$. Thus by the definitions of $G, \psi$ belongs to the family $\Sigma\left(u_{1}, u_{2}\right)=G^{-1}(0) \cap U$, which is of codimension 1 in $C^{k, \alpha}(\partial \Omega, N)$.

Then the separability of $C^{k, \alpha}(\Omega, N)$ with respect to norm $\|\cdot\|_{k, \alpha^{\prime}, 0}$ implies that the open cover $\left\{B\left(u_{1}, u_{2}\right):\left(u_{1}, u_{2}\right) \in \mathcal{C}\right\}$ of $\mathcal{C}$ has a countable subcover $\left\{B\left(u_{1}^{i}, u_{2}^{i}\right):\left(u_{1}^{i}, u_{2}^{i}\right) \in \mathcal{C}, i=1,2,3, \ldots\right\}$. Thus the relevant set of nonunique boundary data is contained in $\cup_{i=1}^{\infty} \Sigma\left(u_{1}^{i}, u_{2}^{i}\right)$, which is of codimension 1 .

Because a subset of $C^{k, \alpha}(\partial \Omega, N)$ of codimension 1 is of first category, we may combine Theorems 6.6 and 6.8 to obtain the following:

6.9. Corollary. The set of all $\varphi \in C^{k, \alpha}(\partial \Omega, N)$ that serves as boundary value of two or more harmonic maps in $C^{k, \alpha}(\Omega, N)$ having the same energy is of first category in $C^{k, \alpha}(\partial \Omega, N)$.

\section{REFERENCES}


[ADN] S. Agmon, A. Douglis and L. Nirenberg, Estimates near the boundary for solution of partial elliptic differential equations satisfying general boundary conditions II. Comm. Pure Appl. Math. 17 (1964), 35-92.

[BA] A. Blades, Stability and uniqueness properties of the equator map from a ball into an ellipsoid, Math. Zeit. (1984)

[BCL] H. Brezis and J.-M. Coron and E. H. Lieb, Harmonic maps with defects, Comm. Math. Phy., 107 (1986) 649-705.

[C] A.P. Calderòn. Uniqueness in the Cauchy problems for partial differential equations. Amer. J. Math. 80 (1958), 16-35.

[CHS] L. Caffarelli, R. Hardt and L. Simon, Minimal surfaces with isolated singularities. Manuscripta Math. 48(1984) 1-18.

[DN] A. Douglis and L. Nirenberg, Interior estimates for elliptic systems of partial differential equations. Comm. Pure Appl. Math. 8.(1955), 503-538.

[EL] J. Eells and L. Lemaire, Another report on harmonic maps, Bull. London Math. Soc. 20(1988), 385-524.

[F] H. Federer, "Geometric Measure Theory", Springer-Verlag, 1969.

[GT] D. Gilbarg and N. Trudinger, "Elliptic Partial Differential Equations of Second Order", Springer-Verlag, New York, Berlin, Heildelberg, 1983.

[HKL] R. Hardt, D. Kinderlehrer and F. H. Lin,Existence and partial regularity of static liquid crystal configurations. Comm. Math. Physics, 105(1986), 547-570.

[HL1] R. Hardt and F.H. Lin, Mappings minimizing the $L^{p}$ norm of the gradient. Comm. Pure Appl. Math. 40(1987), 555-588.

[HL2] R. Hardt and F.H. Lin, Stability of singularities of minimizing harmonic maps. J. Diff. Geom. 29(1987), $113-123$.

[HS] R. Hardt and L. Simon, Area minimizing hypersurfaces with isolated singularities, J. Reine. Angew. Math. 362 (1985), 102-129.

[H] S. Hildebrandt, Harmonic mappings of Riemaniann manifolds, in Lecture Notes in Mathematics 1161, Springer-Verlag, 1985, pp 1-117.

[JK] W. Jäger and H. Kaul, Rotationally symmetric harmonic maps from a ball into a sphere and the regularity problem for weak solutions of elliptic systems. J. Reine Angew. Math. 343 (1983) 146-161.

[J] J. Jost, "Harmonic mappings between Riemannian manifolds", Proc. Centre Math. Analysis, 1983.

[L] S. Lang, "Differential Manifolds", Adison-Wesley, Reading, Massachusetts.

[LF] F.H. Lin, A remark on the map $\frac{x}{|x|}$, Acad. Sci. Paris 305, I (1987), 529-531.

[MS] R. Macintosh and L. Simon, Perturbing away singularities of harmonic maps, Manuscripta Mathematica, 67 (1990) 113-124.

[MF1] F. Morgan, Almost every curve in $\mathbf{R}^{3}$ bounds a unique area minimizing surface. Invent. Math. 45(1978), 253-297.

[MF2] F. Morgan, Measure on space of surfaces. Arch. Rational Mech. Anal. 78(1982), 335-359.

[MF3] F. Morgan, Generic uniqueness for hypersurfaces minimizing the integrand with constant coefficients. Indiana U. Math. J. 30(1981), 29-45.

[MC] C. B. Morrey, Jr., "Multiple Integrals in the Calculus of Variations", Springer-Verlag, 1966.

[ML1] L. Mou, Harmonic maps with prescribed finite singularities. Comm. in P. D. E. Vol. 14, 11(1989), $1509-1540$.

[ML2] L. Mou, Uniqueness of energy minimizing maps for almost all smooth boundary data. Indiana Univ. Math. Jour. Vol. 40, No 1 (1991) 363-392.

[SU1] R. Schoen and K. Uhlenbeck, A regularity theory for harmonic maps. J. Diff. Geom. 17(1982), $307-335$. 
[SL] L. Simon, Isolated singularities for extrema of general variational problems. Lecture Notes in Mathematics 1161, Springer-Verlag, 1985.

[SN1] N. Smale, Minimal surfaces with many isolated singularities Ann. of Math. 130(1989), 603-642.

[SN2] N. Smale, An equivariant construction of minimal surfaces with nontrivial singular sets, Indiana Univ. Math. Jour. 40, No.2 (1991)595-616.

[SN3] N. Smale, Geometric P.D.E.'s with isolated singularities. Preprint.

[SRT] R. T. Smith, The second variation for harmonic mappings, Proc. Amer. Math. Soc. 47 (1975) $229-236$.

[T] M. Taylor, "Pseudodifferential Operators", Princeton University Press, 1981.

[WB] B. White, The space of m-dimensional surfaces that are stationary for a parametric elliptic functional, Indiana Univ. Math. Jour. Vol. 36, 3(1987), 567-602.

*Research partially supported by the National Science Foundation

Mathematics Department, Rice University, Houston, TX 77251

Department of Mathematics, University of Southern California, Los Angeles, CA 90089 